\title{
Experiments on the forced wake of an airfoil
}

\author{
By M. GHARIB AND K. WILLIAMS-STUBER† \\ Department of Applied Mechanics and Engineering Sciences, University of California, \\ San Díego, La Jolla, CA 92093, USA
}

(Received 7 June 1988)

The effect of initial flow conditions on the wake of an airfoil is examined in an experiment which uses the 'strip heater' technique to externally force the airfoil wake. The strip heaters are used to introduce waves into the top and bottom boundary layers of a thin symmetric airfoil which are subsequently amplified and introduced to the wake. The evolution and interaction of the waves in the wake is the primary interest of this study. A linear stability analysis is applied to the mean velocity profiles in order to understand the frequency selection process in the wake. It is seen that the mean velocity profile adjusts itself in order to become more receptive to the forced frequency of oscillation, resulting in the suppression of previously existing frequencies. The amplitude of oscillations in the wake can be controlled by varying the phase relation between two input signals. In this respect, cancellation and enhancement of the oscillations is possible. The linear stability analysis is applied to the cancellation/enhancement flow to verify the level of cancellation achieved. The receptivity of the system to external forcing is established. A substantial reduction in drag is achieved for forcing frequencies near the centre of the receptivity range.

\section{Introduction}

In recent years, there has been considerable interest in the application of feedback/control systems to fluid dynamical systems. For the engineer, external control can be used to improve the characteristics of a system (for example, to reduce the drag or to delay the transition to turbulence). From a researcher's viewpoint the advantages of forcing are multifold. Through the use of external control, a better environment for examining the nature of the system is obtained by establishing a greater number of known parameters. The introduction of a known perturbation to the flow provides a reference for the nature and intensity of the predominant disturbance which precipitates the natural transition (i.e. the amplification and rollup of perturbations into vortices). In addition, the reproducibility of the process under investigation is greatly enhanced.

Presented here is an experimental effort which attempts to understand the nature of the wake system of a thin airfoil in a controlled environment. In general, the wake of bluff bodies is characterized by a region of absolute instability in the near field and one of convective instability further downstream. It is more difficult to modify an absolutely unstable flow with external forcing; thus, it is necessary to remove the absolute instability parameter in order that the external forcing of the convective instability be effective. For this reason, the airfoil geometry was selected because its

† Current address: Department of Mechanical \& Environmental Engineering, University of California, Santa Barbara, CA 93106, USA. 
region of absolute instability is negligible when compared to other geometries. The frequency of oscillation in the wake (the vortex shedding frequency) is controlled through the introduction of an external perturbation. Because the wake is a dynamic system, an active control technique which does not change the overall characteristics of the flow was implemented. The strip-heater technique, which was first introduced by Liepmann, Brown \& Nosenchuck (1982) for flat-plate boundary layers, is used to excite Tollmien-Schlichting waves in the airfoil boundary layers (figure 1). The waves are amplified by the boundary layer and then introduced to the wake. It is the development of the waves in the wake that is the primary interest of this study.

The use of external forcing techniques is not new in the study of free shear layers (which encompasses wakes, jets, and mixing layers). In attempts to understand basic flow dynamics, investigators have relied upon both active and passive control techniques some of which will be described shortly. The first step in designing an experiment which employs external control is the selection of the control technique. External perturbations may be introduced to a flow through either passive or active means. Passive (or steady-state) techniques include the use of passive heating, trip wires and vortex generators (Wygnanski et al. 1979), stationary ribbons (LEBUs), or roughness elements. Active forcing falls into two categories: global techniques and local techniques. Global techniques, such as acoustic forcing (Sato \& Kuriki 1961; Browand 1966; Freymuth 1966; Sato 1970; Miksad 1972, 1973) and variable pressure regulators (Ho \& Huang 1982 ; Roberts 1985), are used to modify the entire free stream (thus modifying the overall characteristics of the system being examined). Local techniques result in a change only to the initial conditions of the system, through the use of moving flaps (Oster \& Wygnanski 1982; Wygnanski, Champagne \& Marasli 1986), vibrating ribbons (Schubauer \& Skramstad 1947; Wygnanski et al. 1979 ; Zaman \& Hussain 1981), or active heating elements (Liepmann et al. 1982; Gharib 1987; Robey 1986). The ability of all but the last technique to provide a system in which clean frequencies can be introduced to the flow is questionable. The mechanical techniques suffer from the obstacle that higher harmonics are often introduced to the system. The present study relies upon the existence of distinct frequencies in order to document the resulting interactions without changing the overall nature of the flow. Accordingly, the strip-heater technique was perceived to be the most suitable method available for achieving the intended goal. To our knowledge, this is the first set of experiments in which the strip-heater technique is used in a flow which contains both signs of vorticity.

The primary goal of most active control systems is to enhance or damp existing perturbations in the flow. A classic flow control experiment by Liepmann \& Nosenchuck (1982) demonstrated that boundary-layer transition could be accelerated or delayed by modifying the phase difference between two strip heaters. In the present study, we present an experiment that, for the first time, applies the same concept to a wake flow. It is seen that both enhancement and cancellation of two waves in the wake is possible through phase variation.

An additional goal of control experiments is to alter the frequency content of the original flow. Sato (1970) and Wygnanski et al. (1986) have both demonstrated the ability to force wakes at frequencies other than the natural vortex shedding frequency. It is known that the introduction of a frequency to a system often results in the suppression of an existing frequency in the system (typically, the natural vortex shedding frequency) (Sato \& Kuriki; Sato; Miksad; Oster \& Wygnanski; Gharib). Previous investigators have conjectured that a change in the mean velocity profile plays a role in the frequency selection process, but there has never been 


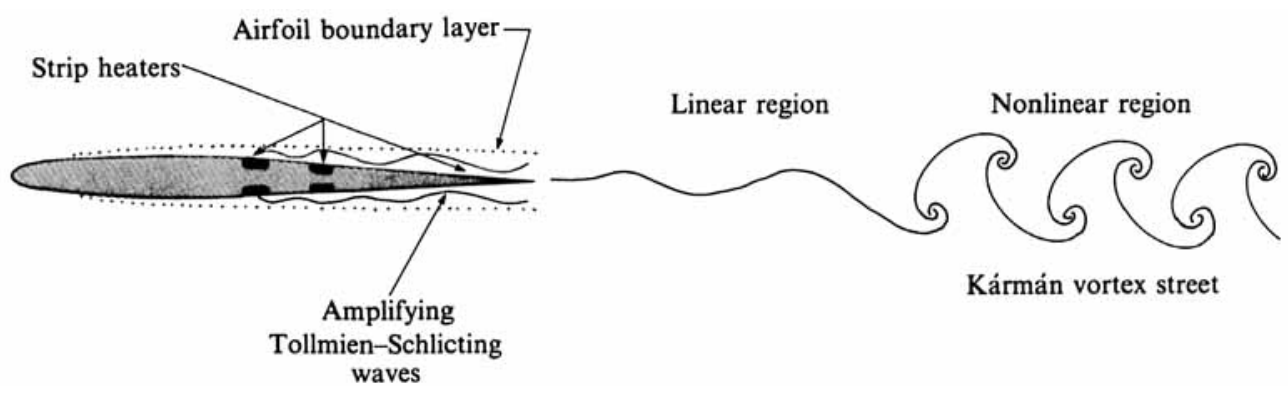

Figure 1. The strip-heater technique for introducing waves into the wake of an airfoil.

concrete evidence to support this hypothesis. It is our belief that an answer to this question is found by examining the stability characteristics of the system.

In examining the stability of a forced flow, many investigators have questioned the validity of comparing the stability of the forced transition to that of the natural transition (Sato \& Kuriki; Browand; Sato; Mattingly \& Criminale 1972; Miksad 1973). Routinely, calculations were performed without verifying the assumption that the forced flow has the same stability characteristics as the natural flow. The present study examines the natural wake and the forced wake through the use of a linear, inviscid stability analysis which has been shown (Freymuth; Ho \& Huang) to be able to predict the initial features of the flow (i.e. the initial vortex shedding frequency) in spite of the nonlinearity of the transition process. In addition, the stability of the cancellation/enhancement profiles is examined in order to verify the degree to which cancellation is obtained. The case of spatially growing waves is considered as it is more applicable to the wake instability process (Mattingly \& Criminale).

Before proceeding with the investigation, it is first necessary to obtain a complete understanding of the receptivity of the system to the forcing frequencies. In this respect, the response of the wake at several points in space for a range of frequencies should be established. A detailed study of the response of the wake to the introduction of external frequencies is presented in the initial sections of this study. Once the wake response is established, a more detailed investigation of the flow characteristics such as velocity fluctuations, drag, and stability is performed.

\section{Experimental details}

The experiments were performed in the UCSD Department of Applied Mechanics \& Engineering Sciences closed-loop water tunnel. The water tunnel has an $8 \mathrm{ft}$. long test section with a $10 \mathrm{in}$. square cross-section. Additional details of the experimental procedures may be found in Stuber (1988).

\subsection{The experimental model}

A thin symmetric airfoil (NACA 63A008) which is characterized by an adverse pressure gradient over much of its surface was mounted $12.7 \mathrm{~cm}(5 \mathrm{in}$.) from the entrance to the test section. The airfoil chord measured $7.6 \mathrm{~cm}(3 \mathrm{in}$.$) ; the thickness$ was $0.64 \mathrm{~cm}(0.25 \mathrm{in}$.); and the span was $24.1 \mathrm{~cm}$ (9.50 in.). Thin stainless steel strips ( 0.002 in. thick, 0.10 in. wide) were flush mounted on the top and bottom surfaces of the airfoil at the $\frac{1}{2}, \frac{2}{3}$, and $\frac{23}{24}$ chord point of the airfoil. Each strip was connected to an independent power supply which was driven by a signal generator. For some of the tests, a phase shifter was included to control the phase relation between the two 
strips. Dye ports were located at the centre of the airfoil on the top and bottom of the leading edge. To visualize the flow, dye was gravity fed to the injection tubes and discharged into the airfoil boundary layers.

\subsection{Operational procedures/reference definitions}

Flow velocities were measured with a single-component laser-Doppler anemometer. A dual-beam forward-scatter configuration was used to measure the downstream component of velocity $(u)$. The velocity time series was low-pass filtered at $10 \mathrm{~Hz}$ using an analog filter. Subsequently, the analog signal was digitized and stored on a MASSCOMP data acquisition system for further processing. The velocity time series was digitized at a rate of 250 samples/s. For power spectra measurements, the digitized traces were resampled at a rate of 25 samples/s corresponding to a minimum Nyquist frequency of $12.5 \mathrm{~Hz}$. The sampling time ranged from $30 \mathrm{~s}$ to $90 \mathrm{~s}$, which was considered adequate to characterize the flow being observed. Real-time power spectra of the velocity signal were obtained on a Hewlett Packard dynamic signal analyser.

Measurements were made at flow speeds from $5 \mathrm{~cm} / \mathrm{s}$ to $12 \mathrm{~cm} / \mathrm{s}$. The Reynolds number based on the model chord length, $c=7.6 \mathrm{~cm}\left(3 \mathrm{in}\right.$.), at $U_{\infty}=11.5 \mathrm{~cm} / \mathrm{s}$ (at which most tests were performed) is 8600 . The origin of the reference system is located at the trailing edge of the airfoil which was positioned with zero angle of attack. The $x$-axis is in the flow direction, the $y$-axis in the cross-stream direction, and the $z$-axis in the spanwise direction. The velocity and length scales incorporated in the analysis of the experiment are $U_{\infty}$ the free-stream velocity, $U_{\mathrm{c}}$ the centreline mean velocity, $u^{\prime}, v^{\prime}$, and $w^{\prime}$ the fluctuation velocities in the $x$-, $y$-, and $z$-directions, respectively, $\delta$ the experimental wake width measured from the wake centreline $(y=0)$ to the point where $u=0.99 U_{\infty}, Y_{\text {peak }}$ the $y$-distance between the two maxima of the fluctuation velocity profile, and $b$ a theoretical half-wake width used in curve fitting such that $u(y / b=1)=\frac{1}{2}\left(U_{\infty}+U_{\mathrm{c}}\right)$. When referring to the fluctuation velocities, $\left(u^{\prime 2}\right)^{\frac{1}{2}}$ will indicate the r.m.s. velocity, and $\overline{u^{\prime 2}}$ will denote time average of the fluctuation value squared. For natural flow (unforced) at $x / c=1, \delta=0.515 \mathrm{~cm}$ results in a Reynolds number based on $\delta\left(R e_{\delta}\right)$ of 590 .

\subsection{Strip-heater technique}

Perturbations are introduced to the boundary layer of the airfoil through the use of strip heaters. Metal strips are flush mounted on each side of the airfoil. A sinusoidal electric current is applied to the strip heater which results in a local change of the viscosity of the fluid in contact with the strip. The temperature difference between the strip and the free stream was experimentally determined to be on the order of $0.28^{\circ} \mathrm{C}$ which implies that buoyancy effects are negligible. For periodic heating, the sinusoidal disturbance is convected downstream by the mean flow velocity.

From linear boundary-layer stability theory, it is known that only a finite range of perturbation frequencies will be amplified by the boundary layer. It is this range of frequencies that is forced using the strip-heater technique. Linear stability also predicts that an adverse pressure gradient (which exists for the chosen airfoil) will improve the receptivity of the airfoil bounday layer to the external forcing.

Owing to the quadratic joule heating effect, it is necessary to generate an input signal at one-half the desired output frequency. In addition, each frequency must be input on a separate strip. 


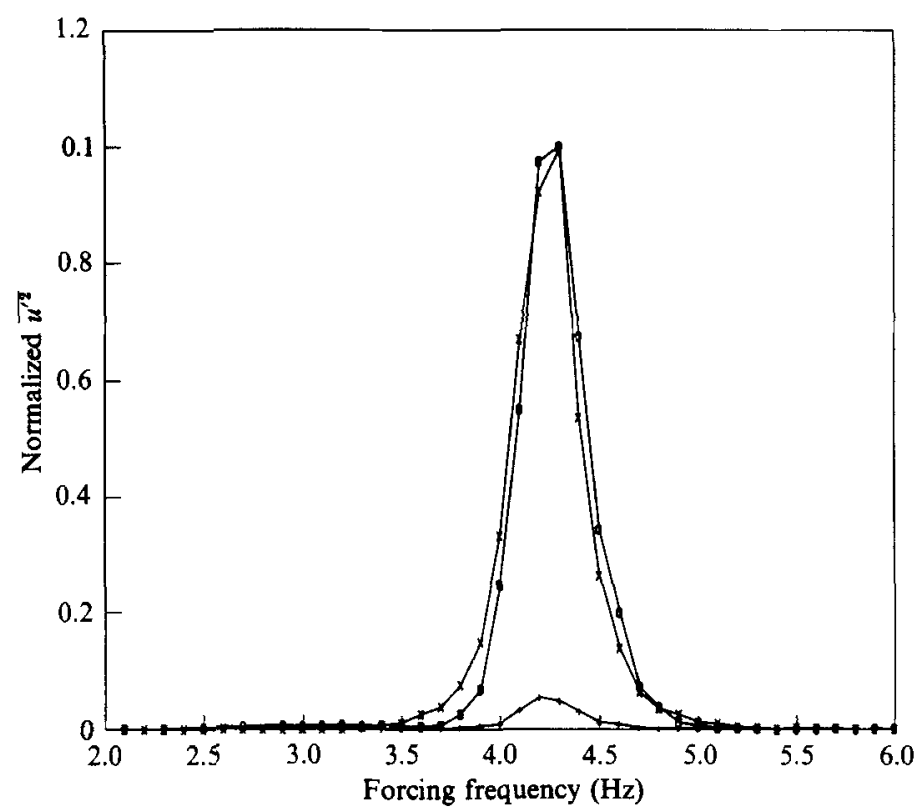

Figure 2. Receptivity curves for three strip-heater positions $(O$, front; $x$, middle; + , trailing edge), at $x / c=\frac{1}{3}$ and $y=\delta_{n}$.

\section{Results and discussion}

\subsection{Receptivity}

The first step in examining a forced wake is to introduce a single frequency into the flow and to monitor the wake response. By sweeping over a range of frequencies near the natural vortex shedding frequency, a receptivity curve is constructed by measuring the response in a narrow band (bandwidth $=0.1 \mathrm{~Hz}$ ) centred at the forcing frequency. This power is a measure of the bandwidth $\overline{u^{\prime 2}}$ for the frequency. The sum of the power in all frequencies is equal to $\left[\left(u^{\prime 2}\right)^{\frac{1}{2}}\right]^{2}$.

The input power to the strip is held constant for each curve which is measured at $y=\delta$ of the natural wake, $\delta_{\mathrm{n}}$. The receptivity curves are all normalized by the maximum value obtained for each set of measurements. The effect of placement of the strip heaters is examined by measuring the receptivity at $x / c=\frac{1}{3}$. It is seen (figure 2) that strips 1 and 2 (mounted at the $\frac{1}{2}$ and $\frac{2}{3}$ chord points, respectively) generate similar results in the flow, whereas strip 3 (placed at the trailing edge) has little effect on the flow. The results for strip 3, which introduces the perturbation directly to the wake with no time for boundary-layer amplification, support the concept introduced by Gharib (1987) that the boundary-layer amplification is essential for the strip-heater technique to be effective in forcing shear flows. The most amplified frequency is $4.2 \mathrm{~Hz}$, which is very close to the natural vortex shedding frequency.

The next set of tests considers the role of the forcing power level using the top middle strip (figure 3). As the power increases, the range of amplified frequencies expands and a greater maximum amplification value is attained. For a range of frequencies near the natural frequency, the vortex shedding frequency tracks or 'locks' onto the forcing frequency. Frequencies for which this occurs are considered to be in the 'locking range'. A formal definition of locking range was established: 


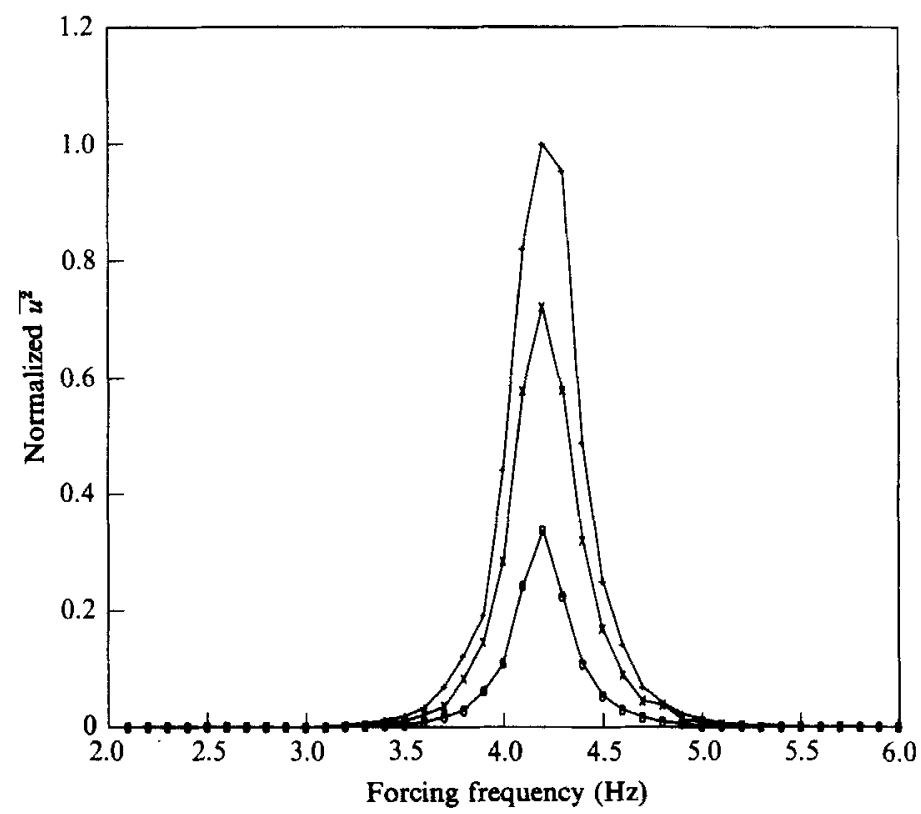

Figure 3. Receptivity curves for three power levels $(O, 5.4 \mathrm{~W} ; \times, 9.8 \mathrm{~W} ;+, 14.1 \mathrm{~W})$, at $x / c=1$ and $y=\delta_{\mathrm{n}}$.

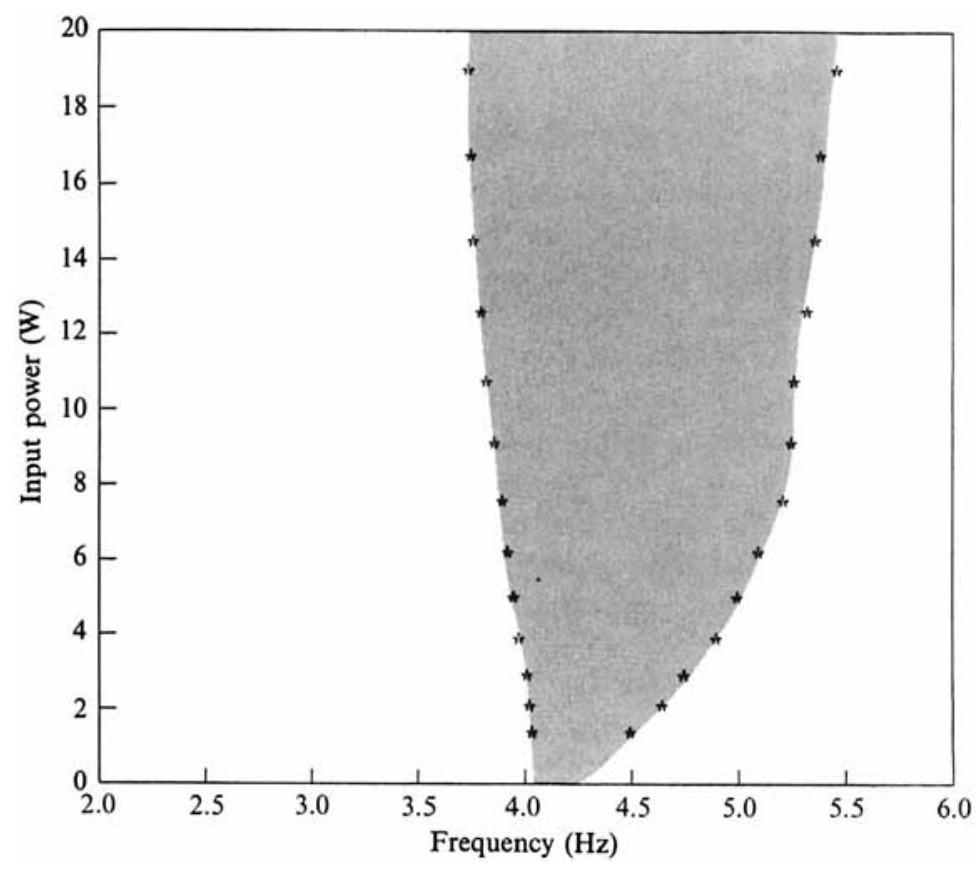

Figure 4. The locking range at $x / c=3$ and $y=\delta_{\mathrm{n}}$.

locking resulted when the amplitude of the bandwidth $\overline{u^{\prime 2}}$ for the forcing frequency exceeded that of the natural by one order of magnitude. As the power of forcing increases, a greater number of frequencies are included in the locking range (figure 4). To better understand the concept of locking, power spectra and time series of a natural, unlocked, and locked flow are presented in figures 5-7. Notice the irregular 

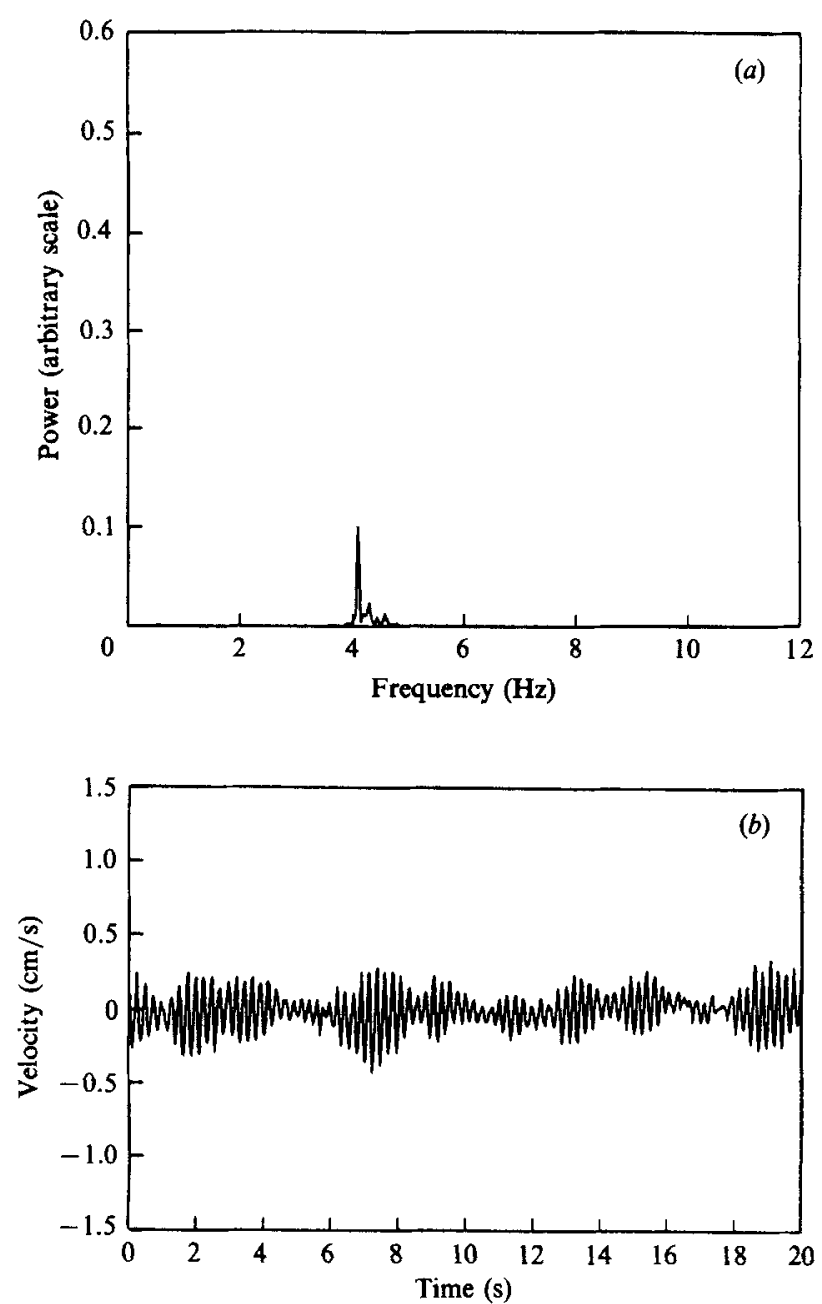

Figure 5. (a) Power spectrum and (b) time series for the natural wake at $x / c=3$ and $y=\delta_{\mathrm{n}}$.

time series and the broadened peak in the power spectra for the natural (figure 5) and unlocked (figure 6) flows. The locked case (figure 7), on the other hand, has a uniform time series and has a narrow, strong peak in the power spectrum.

A major concern of investigators who introduce localized disturbances to a flow is whether the natural symmetry of the natural wake profile is preserved. To verify the uniformity of the forcing technique, two receptivity curves were measured at the $y$ location of the peaks at the natural r.m.s. $\left(u^{\prime 2}\right)^{\frac{1}{2}}$ profile at $x / c=3$ (allowing the flow a chance to develop). The similarity of the measurements (figure 8) attests to the fact that the side of the airfoil used for forcing does not affect the resulting flow and that natural symmetry in the wake is preserved. The asymmetry of the two curves when compared to the $x / c=1$ measurements is attributed to the fact (Mattingly \& Criminale) that as the wake develops downstream it widens, allowing longer wavelength (lower frequency) perturbations to become more dominant. This property manifests itself in that, within the range of amplified frequencies, the lower frequencies in that range have higher amplification rates.

Up to this point, only one strip heater was used to force the flow. An additional 

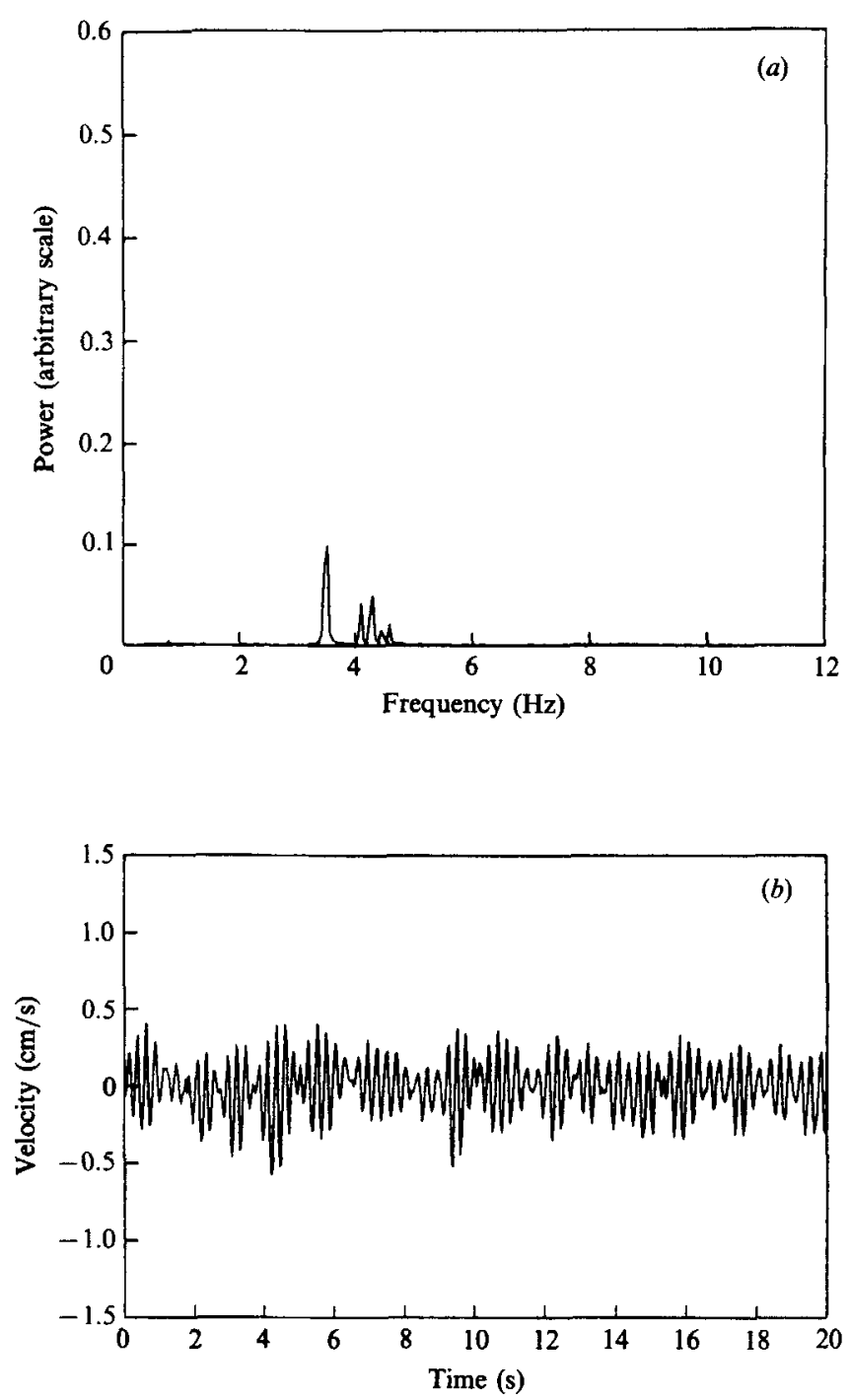

Figure 6. (a) Power spectrum and (b) time series for the unlocked wake at $x / c=3$ and $y=\delta_{\mathrm{n}}$ (arbitrary scale is the same as in figure 5).

receptivity curve (figure 9 ) was constructed at $x / c=3$ for the case where a single frequency was introduced to the flow through the use of two strip heaters operating to produce disturbances which are asymmetric about the wake centreline (enhancing each other). The result is an increase in the range of amplified frequencies while retaining the same general shape of receptivity as the one-sided case. From the results presented so far, the working area for the remainder of the experiments is defined.

\subsection{Cancellation/enhancement experiment}

To better comprehend the wake dynamics, it is useful to learn how to alter them for example, by accelerating or delaying the wave amplification process. Owing to the unstable nature of wakes, it is quite easy to precipitate this transition process. It is much more difficult to delay transition in an unstable system, particularly without use of a feedback system. In the current investigation, one amplified wave 

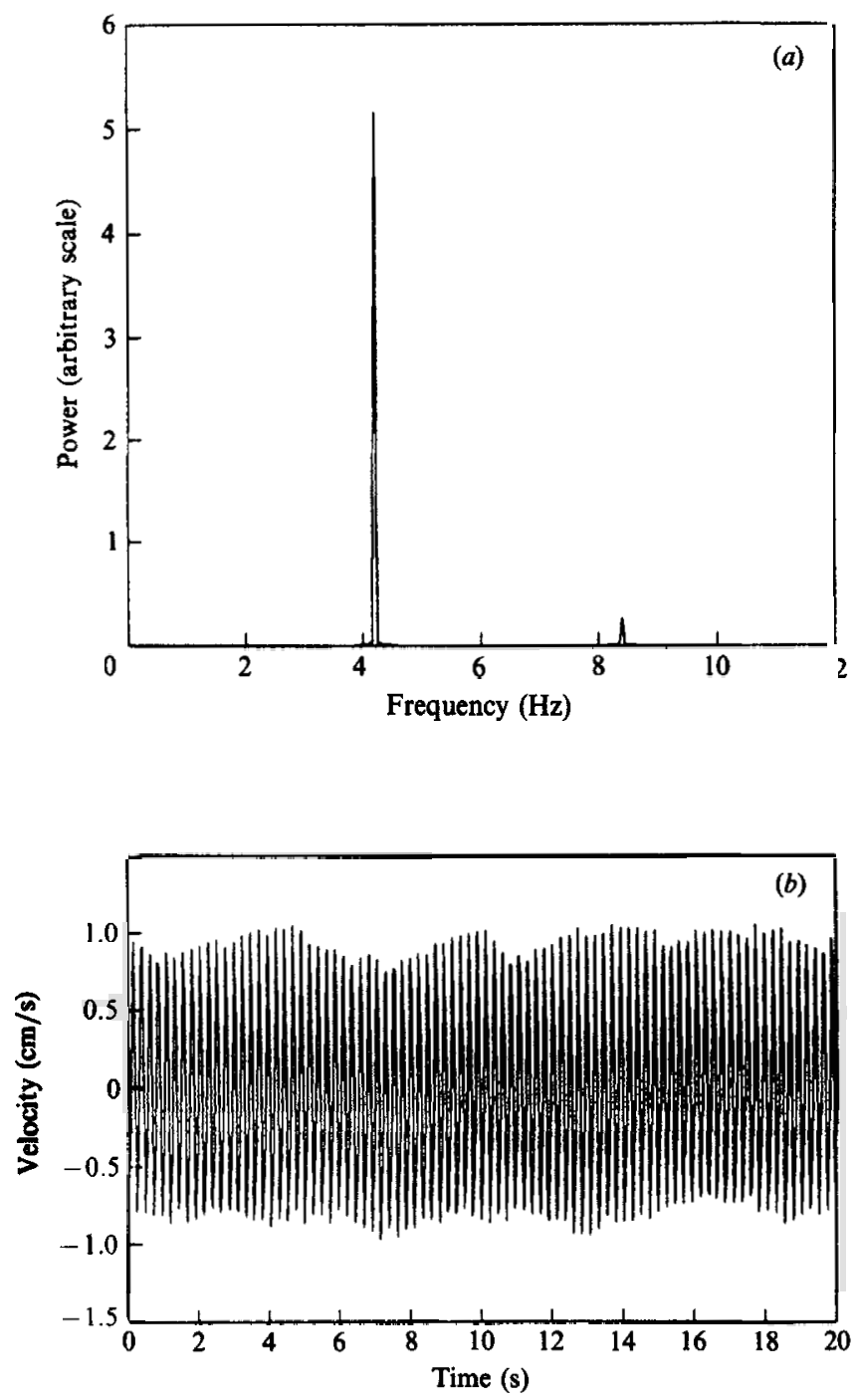

Figure 7. (a) Power spectrum and (b) time series for the locked wake at $x / c=3$ and $y=\delta_{\mathrm{n}}$ (arbitrary scale is the same as in figure 5).

is cancelled or reinforced through the introduction of a second wave by varying the phase difference between the two. The goal of the cancellation is to return the wake to its original (unforced) state. To pursue this notion, a set of measurements were performed in which two strip heaters (one on each side of the airfoil) were operated at the same frequency. The placement of the strips on separate sides of the airfoil ensures that the results obtained are attributed to the wake dynamics, and not to interactions in the airfoil boundary layers. For the first forcing configuration, the strip heaters were operated at the natural shedding frequency to produce perturbations which were asymmetric about the wake centreline, resulting in a reinforcement of the fluctuations. The second case was obtained when the strip heaters were operated in a symmetric configuration, resulting in a cancellation of the two inputs when they meet at the trailing edge. From this point forward, the enhancement case will be referred to as the asymmetric case, and the cancellation 


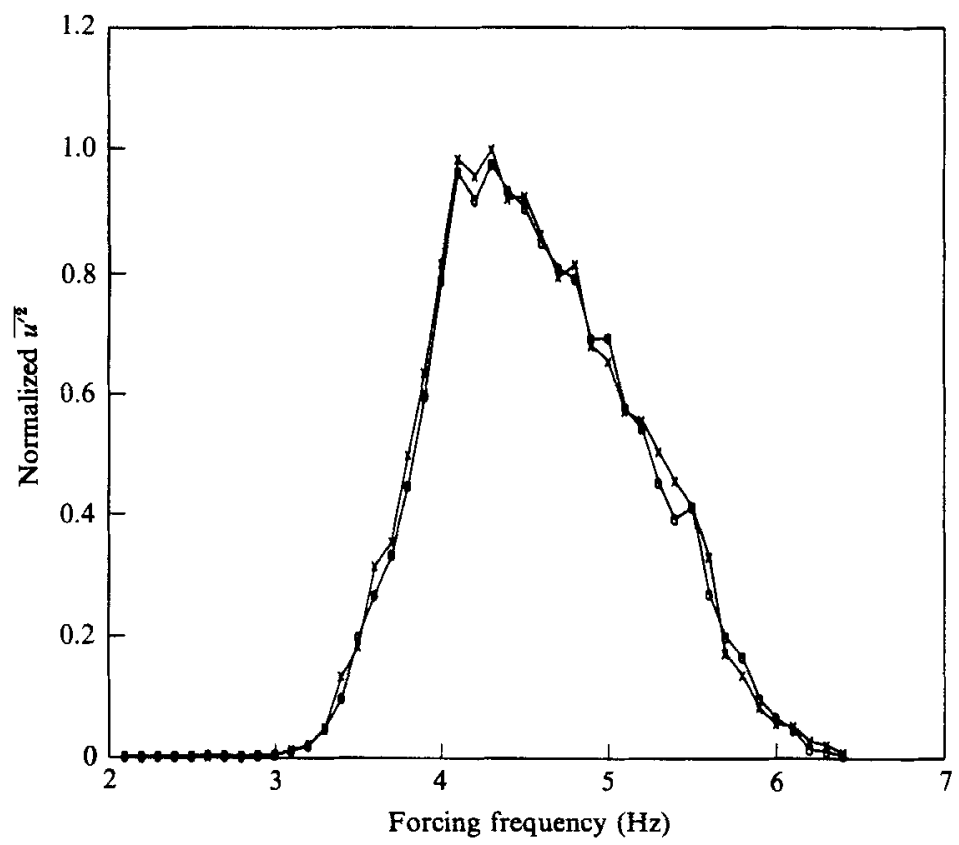

FIGURE 8. Receptivity curves at two points symmetrically above $(O)$ and below $(x)$ the wake centreline, at $x / c=3$ and $y= \pm \frac{1}{2} Y_{\text {peak }}$.

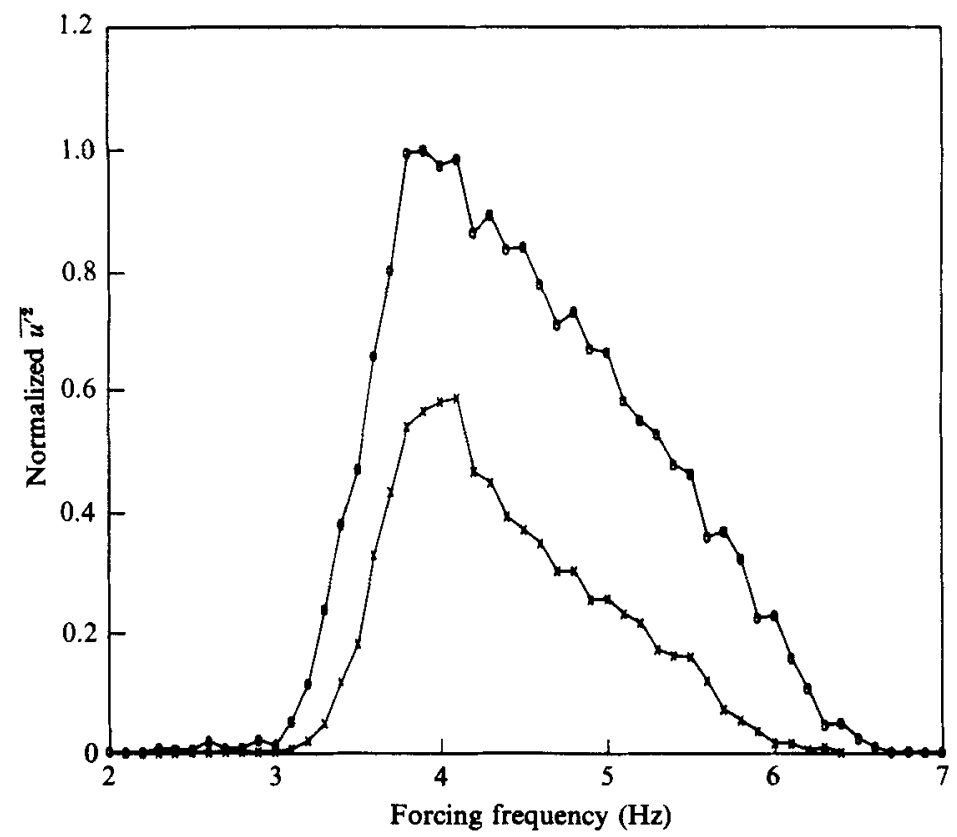

FIGURe 9. Receptivity curve for one-sided $(x)$ and two-sided (O) (asymmetric) forcing at $x / c=3$ and $y=\delta_{\mathrm{n}}$.

case will be called the symmetric case. Time series and associated power spectra for the natural, asymmetric, and symmetric forced wakes (measured at $y=\delta$ of the natural wake at $x / c=1$ ) are presented in figures 10,11 and 12 . It is seen that the peak amplitudes of the natural and symmetric cases are very similar. 

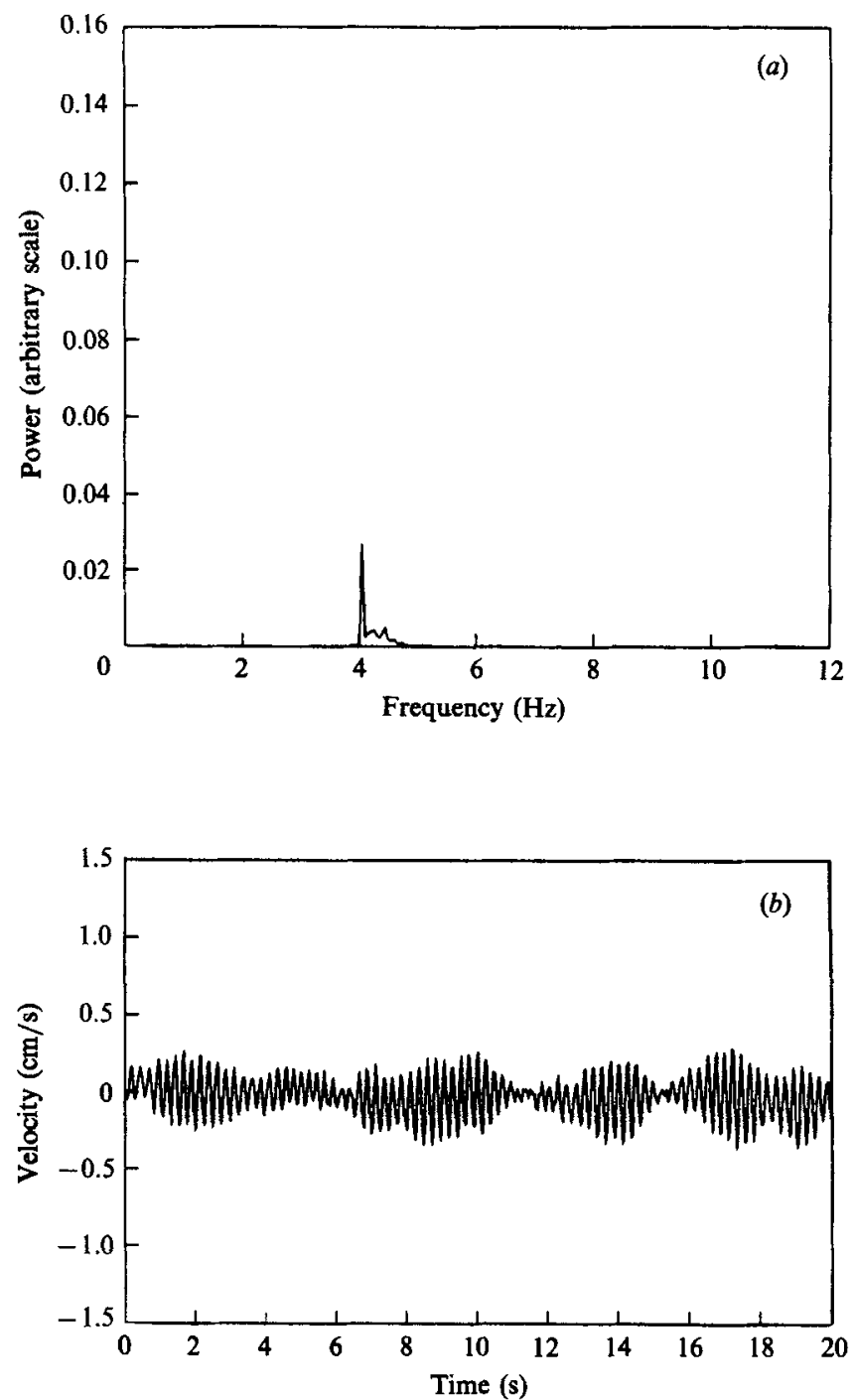

Figure 10. (a) Power spectrum and (b) time series for the natural wake at $x / c=1$ and $y=\delta_{\mathrm{n}}$.

The mean and fluctuating velocity profiles for the asymmetric and symmetric cases are presented in figures 13 and 14, respectively. The natural profile is indicated with a solid line. The peak fluctuations in the enhanced case are $100 \%$ greater than in the natural case. The maximum cancelled fluctuations are within $22 \%$ of the natural case. The mean profile for the symmetric forcing is nearly identical to the natural profile.

With such large variations occurring in the velocity profiles, it is certain that variations of the drag coefficient must occur. To pursue this idea, a control volume analysis was applied to the velocity profiles. Continuity measurements indicated that the mean velocity component in the $y$-direction (which was not measured with the existing LDV arrangement) was too large to ignore at the downstream measuring station $(x / c=3)$. Thus, the momentum analysis was applied to the profiles at $x / c=1$ where negligible cross-stream flow existed. The drag analysis assumed no 

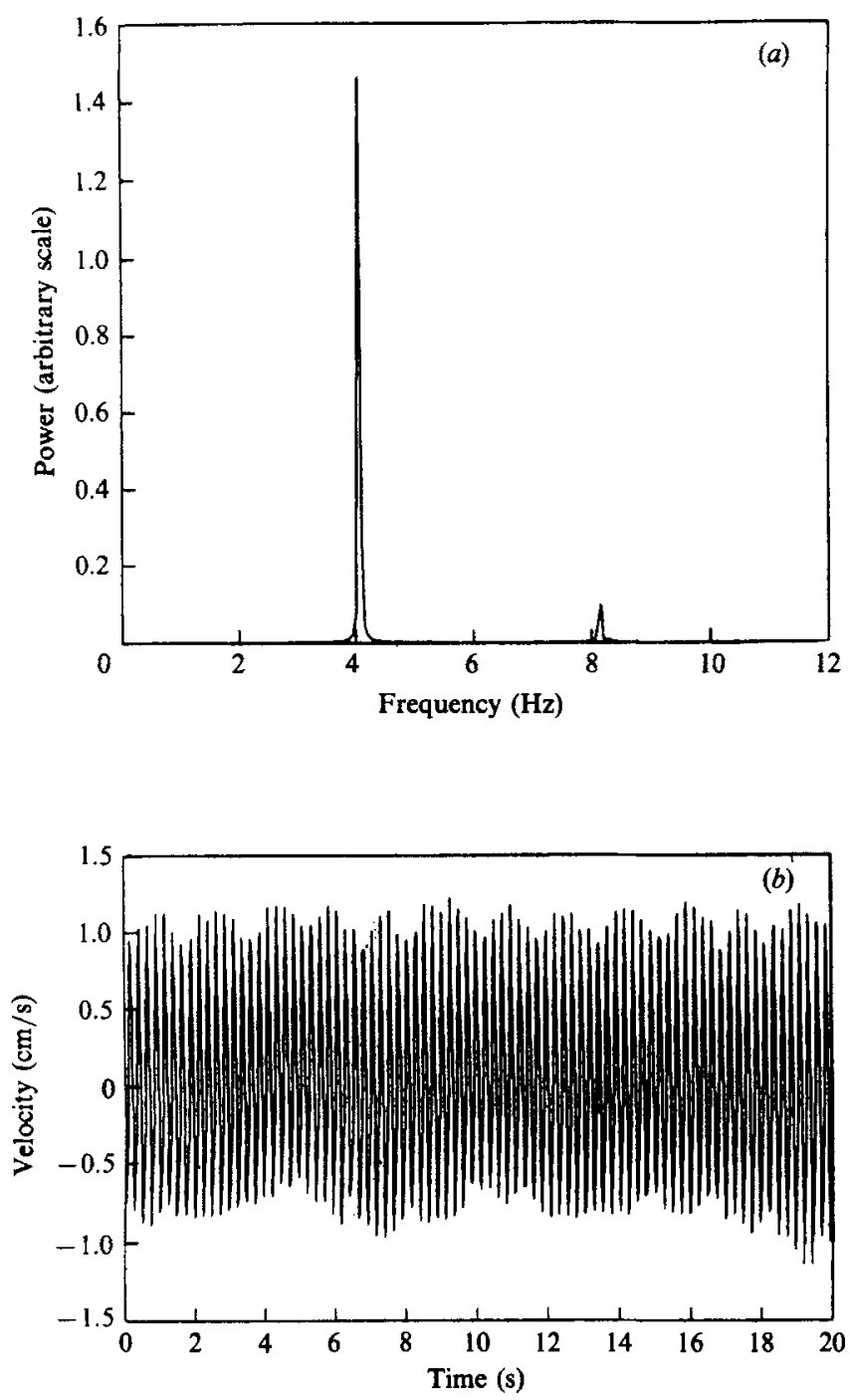

Fraure 11. (a) Power spectrum and $(b)$ time series for asymmetric forcing at $x / c=1$ and $y=\delta_{\mathrm{n}}$ (arbitrary scale is the same as in figure 10).

mean flow in the $y$-and $z$-direction. The fluctuations in the $y$-direction $\left(\overline{v^{\prime 2}}\right)$ and $z$ direction $\left(\overline{w^{\prime 2}}\right)$ were assumed to be of the same order of magnitude as $\overline{u^{\prime 2}}$.

A reduction in drag of $30 \%$ is achieved in the enhanced case, whereas little difference is seen for the cancelled case. The cancellation/enhancement drag results allow us to exclude skin-friction changes as a source of drag reduction because it is assumed that the skin friction on each surface is the same for both asymmetric and symmetric forcing when the frequency is held constant. For the strip-heater experiment, Liepmann et al. (1982) showed that the amplifying waves could be thought of as effective sinusoidal surface displacements. At the trailing edge of our airfoil, this displacement effect (now amplified) could be considered analogous to a flapping of the trailing edge in ways similar to the flapping airfoil of Koochesfahani (1987). It is this effective flapping mechanism, which generates thrust, which results in the drag reduction. Koochesfahani observed similar drag reduction; however, the 

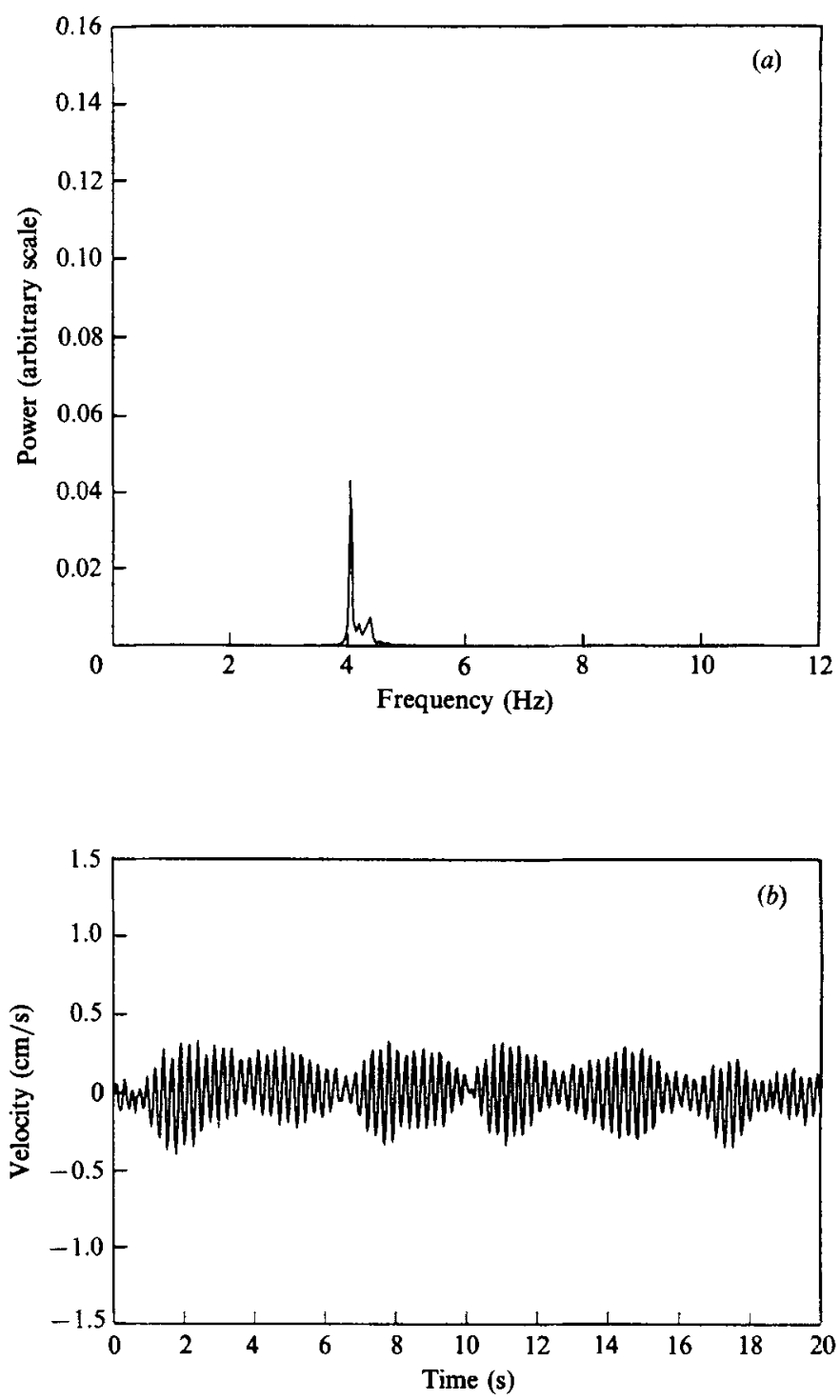

Figure 12. (a) Power spectrum and $(b)$ time series for symmetric forcing at $x / c=1$ and $y=\delta_{\mathrm{n}}$ (arbitrary scale is the same as in figure 10 ).

amplitude of the airfoil oscillations in that work was significantly larger than the level of forcing in the current investigation.

Flow visualization of the natural, asymmetric, and symmetric cases (for a lower velocity case - figure 15) demonstrates an accelerated transition for the asymmetric case. The symmetric case is similar to the natural case, particularly in the near-wake region. Eventually natural instabilities will amplify even in the cancelled case.

The cancellation above was achieved by forcing the flow at the natural frequency. It would be interesting to know how the cancellation procedure works with a nonnatural frequency. To examine this concept, a flow with a natural frequency of $1.875 \mathrm{~Hz}$ was forced at $2.2 \mathrm{~Hz}$. It can be seen from the power spectra (figure 16) and corresponding time series (figure 17 ) of natural $(a)$, asymmetric $(b)$, and symmetric 

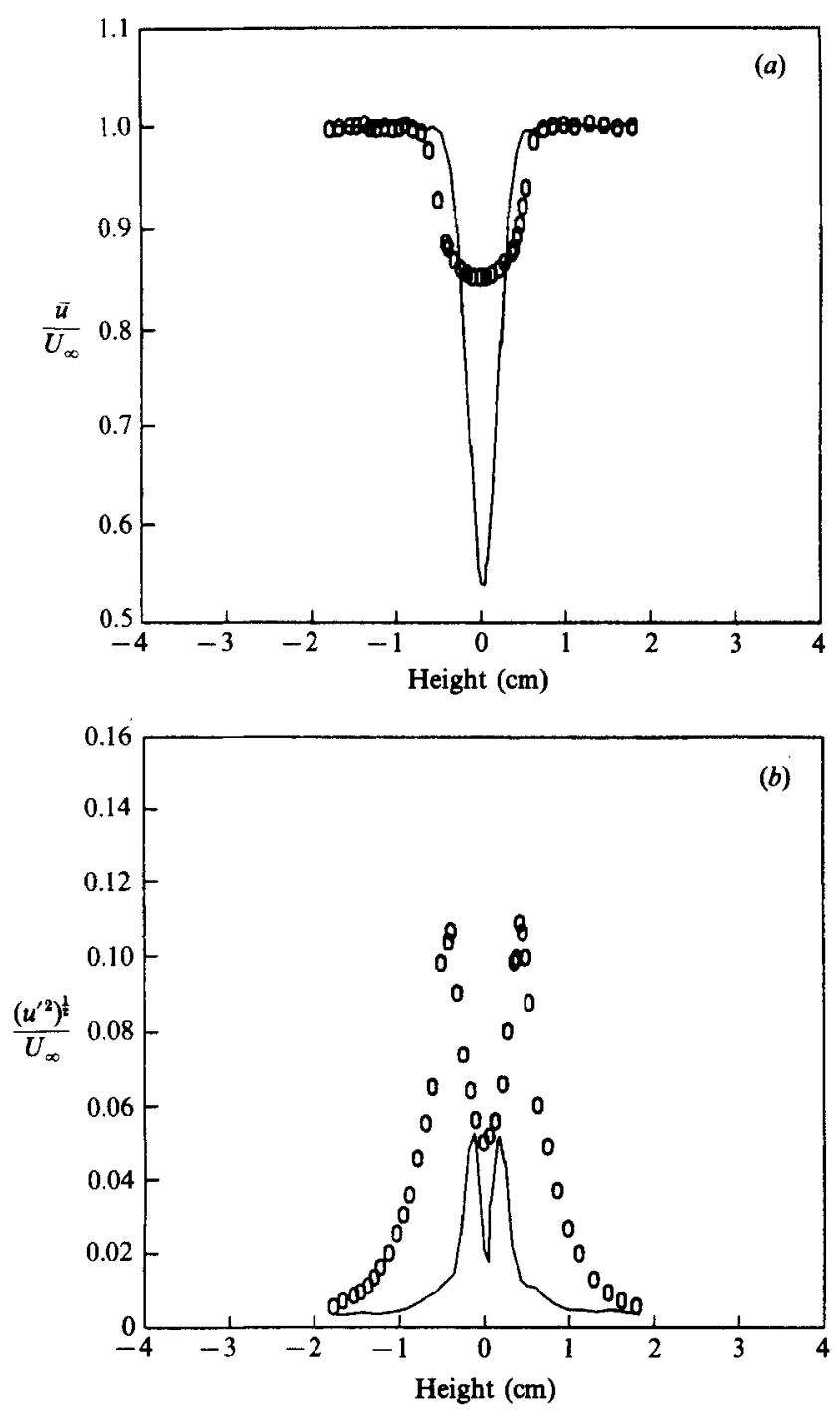

Figure 13. Velocity profiles of the asymmetric case $(0)$ compared to the unforced case $(-)$ at $x / c=1:(a)$ mean velocity; (b) r.m.s. velocity.

(c) forcing that the natural frequency is suppressed by the asymmetric forcing. When forced symmetrically, the natural frequency reappears, supporting the conjecture that symmetric forcing re-establishes the original state of the wake. Verification of this hypothesis will be presented as part of the stability analysis.

\subsection{Velocity profile analysis}

The cancellation experiment focuses primarily on forcing the flow at the natural frequency. However, given a range of amplified frequencies, it is possible to study the mean and fluctuation (r.m.s.) velocity profiles for several different forcing frequencies in the range. To investigate the effects of forcing in more depth, velocity profiles were measured at $x / c=1$ (station I) and at $x / c=3$ (station II) for several frequencies at a fixed power level $(8 \mathrm{~W})$. The normalized centreline velocity $\left(U_{\mathrm{c}} / U_{\infty}\right)$, wake width 

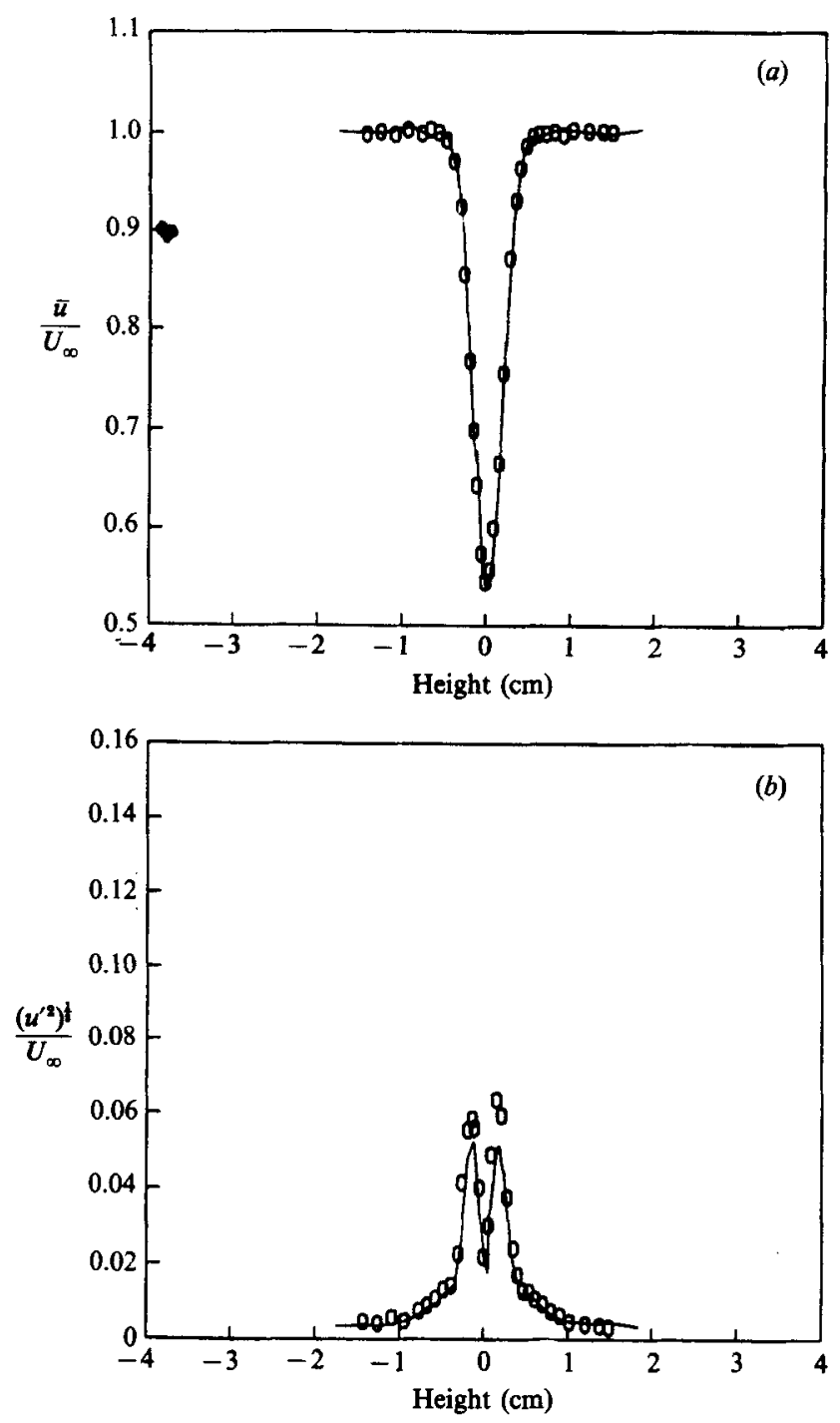

Figure 14. Velocity profiles of the symmetric case $(O)$ compared to unforced case (-) at $x / c=1:(a)$ mean velocity; (b) r.m.s. velocity.

$(\delta)$, and distance between the two maxima in the r.m.s. profile $\left(Y_{\text {peak }}\right)$ were calculated from the profiles. The results, scaled by the values of the unforced case, are presented in figures 18-20. The station I measurements (marked with an open circle) show the most variation: the mean centreline velocity increases for forcing cases near the natural vortex shedding frequency, whereas little change is seen relative to the unforced case when frequencies away from the natural are introduced. Likewise, $\delta$ and $Y_{\text {peak }}$ are greater for frequencies near the centre of the receptivity range, which indicates that a transition is occurring inside the wake. Considering the station II measurements, it is seen that the mean centreline velocity compared to the unforced case is relatively uniform with slightly higher values near the lower frequencies. $\delta$ and $Y_{\text {peak }}$ vary less than the measurements at station $I$. Larger values are seen for lowfrequency forcing, particularly for the wake width measurements. Given that a wider 

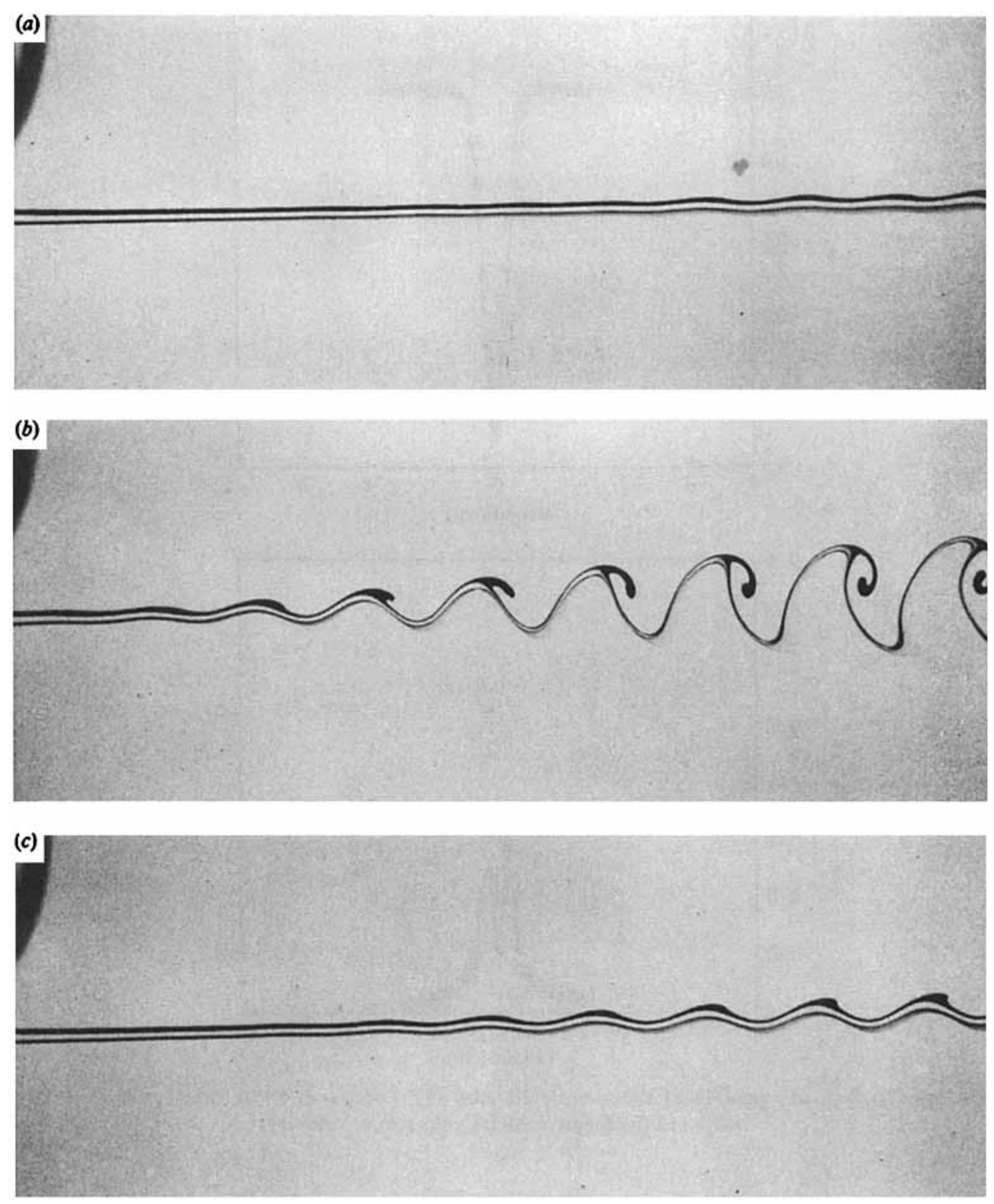

Figure 15. Flow visualization of cancellation/enhancement flows (flow is from left to right, trailing edge is just off the left side of the figure - the right edge corresponds to $\approx x / c=4):(a)$ natural flow; (b) asymmetric forcing; (c) symmetric forcing.

wake can support longer-wavelength oscillations, the shift in maxima towards lower frequencies is expected.

To demonstrate the extreme nature of strongly versus weakly forced wakes (at $x / c=1$ ) compared to the unforced flow, the mean and fluctuating velocity profiles for forcing at $4.2 \mathrm{~Hz}$ and $5.5 \mathrm{~Hz}$ are presented in figures 21 and 22 , respectively. The solid line in the plots represents the natural wake. In the mean profile for the locked case of $4.2 \mathrm{~Hz}$, the deficit decreases dramatically, and the wake widens. The 

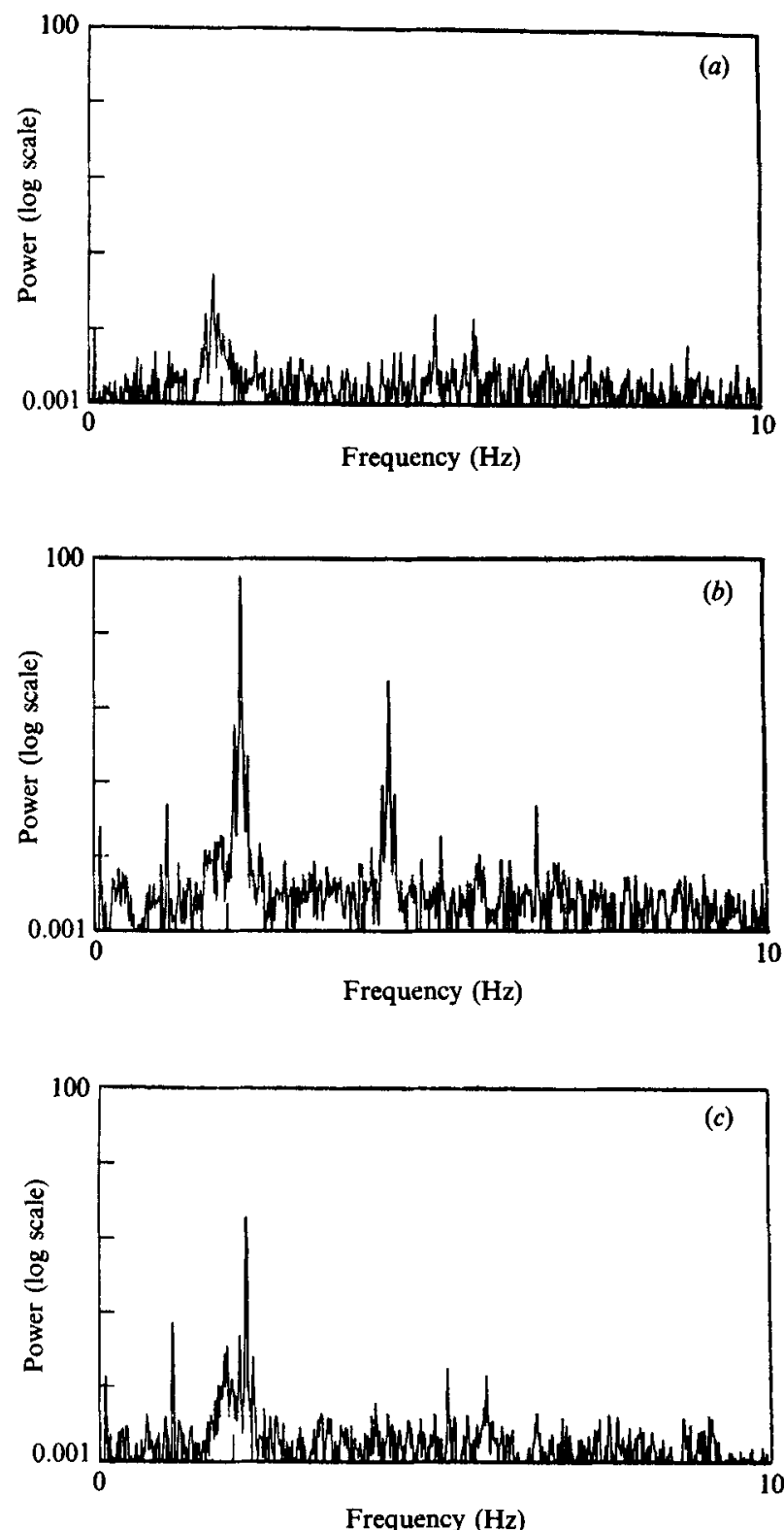

Figure 16. Power spectra for non-natural frequency cancellation cases $\left(f_{\mathrm{n}}=1.875 \mathrm{Ha}\right.$, flow forced at $2.2 \mathrm{~Hz}, U_{\infty}=5.75 \mathrm{~cm} / \mathrm{s}$, at $x / c=1$ and $\left.y=\delta_{\mathrm{n}}\right):(a)$ natural wake; $(b)$ asymmetric forcing; $(c)$ symmetric forcing.

maximum fluctuation value is two and a half times greater for the forced case. The widening of the wake is demonstrated through the increase in the distance between the fluctuation peaks. In contrast, little difference is seen for the mean and r.m.s. velocity profiles for the unlocked case forced at $5.5 \mathrm{~Hz}$ when compared to the natural case. The change in profiles is similar to the changes seen for the cancellation/enhancement cases (figures 13 and 14).

The r.m.s. profiles provide information pertaining to fluctuations at all frequencies. It is interesting to isolate the fluctuations due to the forcing frequency and its 

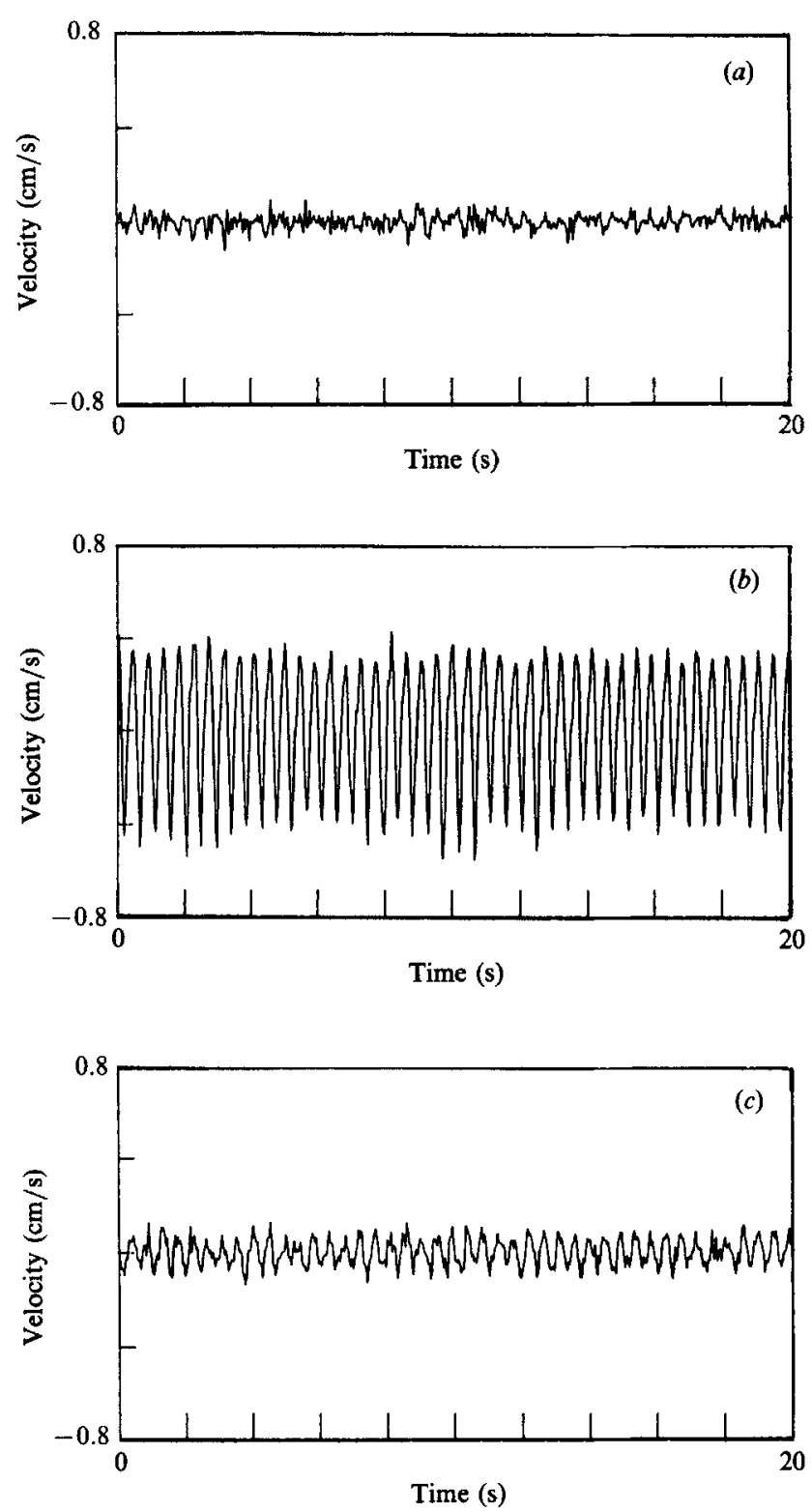

Figure 17. Time series corresponding to power spectra in figure $6:(a)$ natural wake; $(b)$ asymmetric forcing; (c) symmetric forcing.

harmonic from the remaining frequencies. Using the technique for measuring the bandwidth $\overline{u^{\prime 2}}$ for the forcing frequency as presented in $\$ 3.1$ above, bandwidth profiles can be obtained to determine the contribution of the forcing frequency to the overall fluctuation profile. In the following profiles (figures $23-25$ ), the total $\overline{u^{\prime 2}}$ is indicated with a solid line. The plots are normalized by the maximum value of the total $\overline{u^{\prime 2}}$ profile. The contribution to the fluctuations due to the forcing frequency is indicated with a cross. The contribution from the second harmonic of the forcing frequency is denoted with a circle. For the locked case $(4.2 \mathrm{~Hz}$ ) (figure 23 ) the forcing frequency is the primary source of fluctuations in the two peaks, while the second 


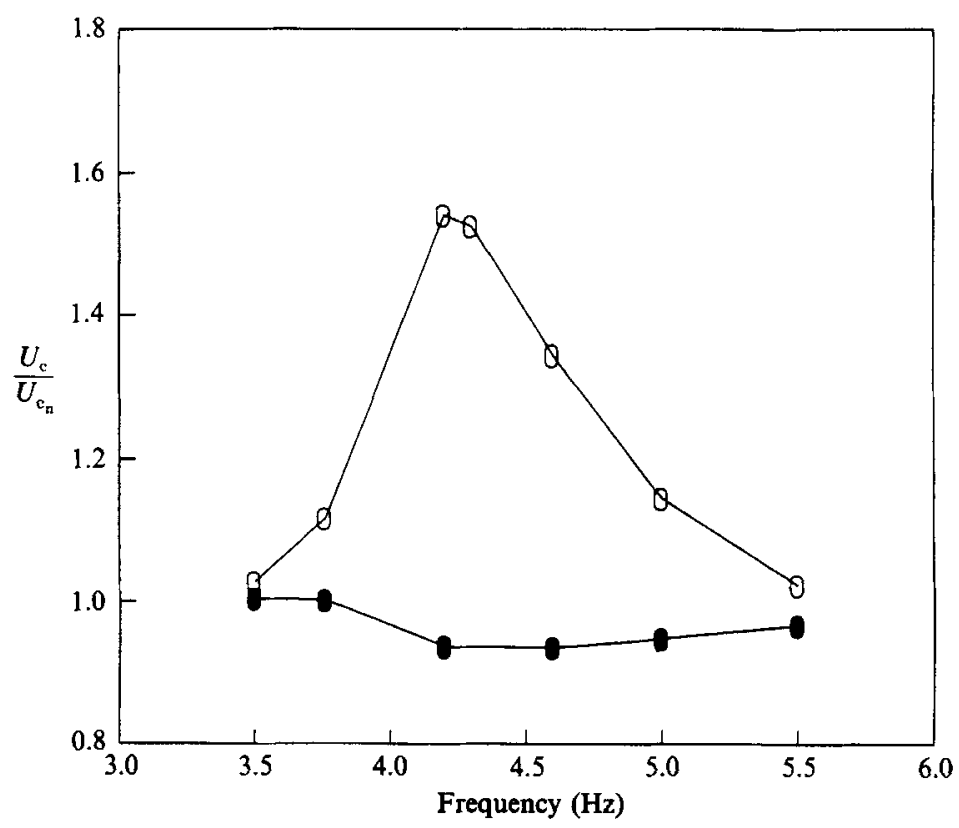

Figure 18. Effect of forcing on the centreline mean velocity compared to the unforced case: $\mathrm{O}, x / c=1 ; 0, x / c=3$.

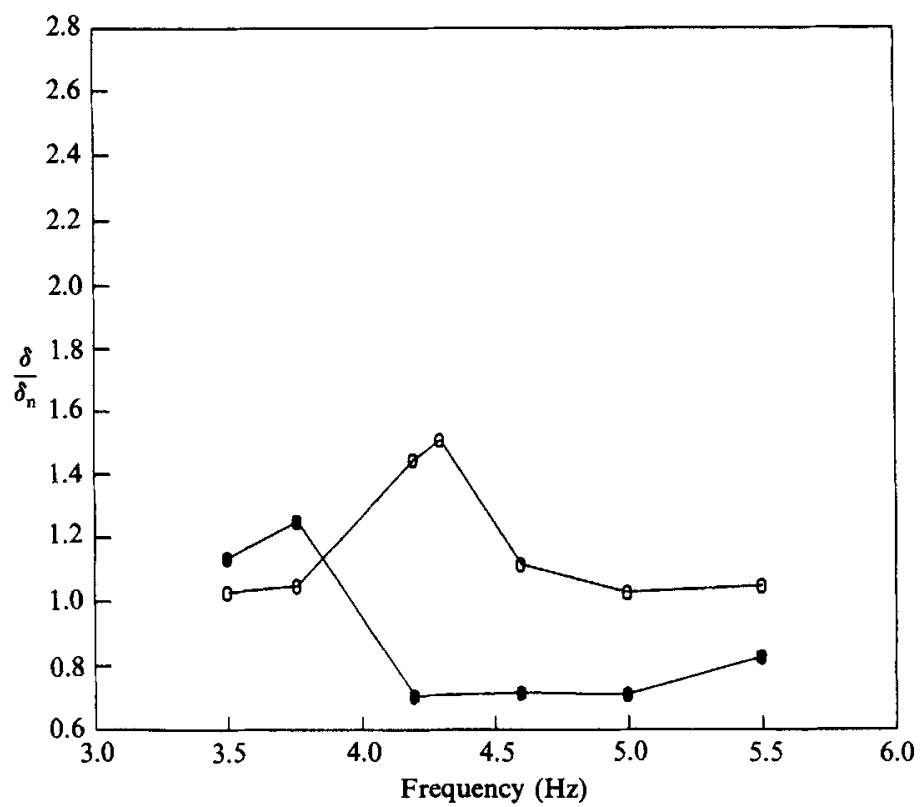

FraUre 19. Effect of forcing on the wake width $(\delta)$ compared to the unforced case: $O, x / c=1$; O, $x / c=3$. 


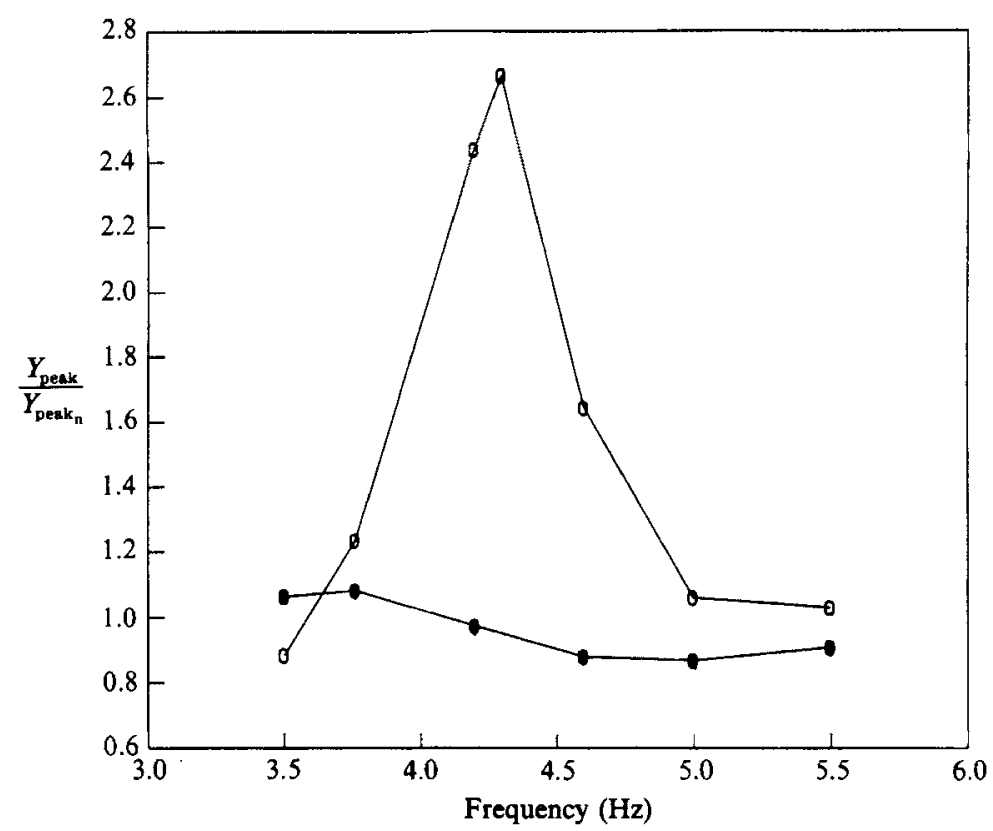

Figure 20. Effect of forcing on the distance between peaks of $\left(u^{\prime 2}\right)^{\frac{1}{2}}$ profile compared to the unforced case: $O, x / c=1 ; 0, x / c=3$.

harmonic dominates the centreline. These results are consistent with the findings of other researchers (Sato \& Kuriki; Sato; Wygnanski et al. 1986). The forcing frequency and its second harmonic are the dominant contributors to the fluctuations in the locked case. In contrast, no contribution from the second harmonic is seen for the cases of unlocked forcing $(5.5 \mathrm{~Hz})$ and the natural wake (figures 24 and 25 , respectively). In addition, the primary frequency in the flow $(5.5 \mathrm{~Hz}$ or the natural frequency) is not the sole contributor to the fluctuations. There are additional frequencies in the flow with significant energy content.

The results of a drag analysis show (figure 26) that profiles of flows forced near the centre of receptivity exhibit a $25 \%$ decrease in drag. As the forcing frequency moves to the edges of the receptivity range, the drag coefficient approaches that of unforced case. Calculations from profiles measured at $x / c=0.15$ show a drag reduction of $10 \%$ for forcing frequencies near the natural frequency. Once again, the drag reduction can be explained by the effective flapping mechanism proposed for the cancellation/enhancement experiment. The smaller drag reduction for the singlesided case results because the effective amplitude of flapping for the single-sided case is lower than the double-sided asymmetric case.

\subsection{Stability analysis}

A fundamental question that has not yet been answered is why the primary frequency in the flow can be shifted to a new frequency in the presence of external forcing. It was conjectured by some investigators (Sato \& Kuriki; Sato; Miksad 1973; Gharib) that changes to the mean profile as a result of forcing are the reason that one sees suppression of one frequency in the presence of a second forced frequency. An example of such behaviour is presented here. The initial stage is characterized by the existence of a single frequency $\left(f_{0}\right)$ in the system (the natural 

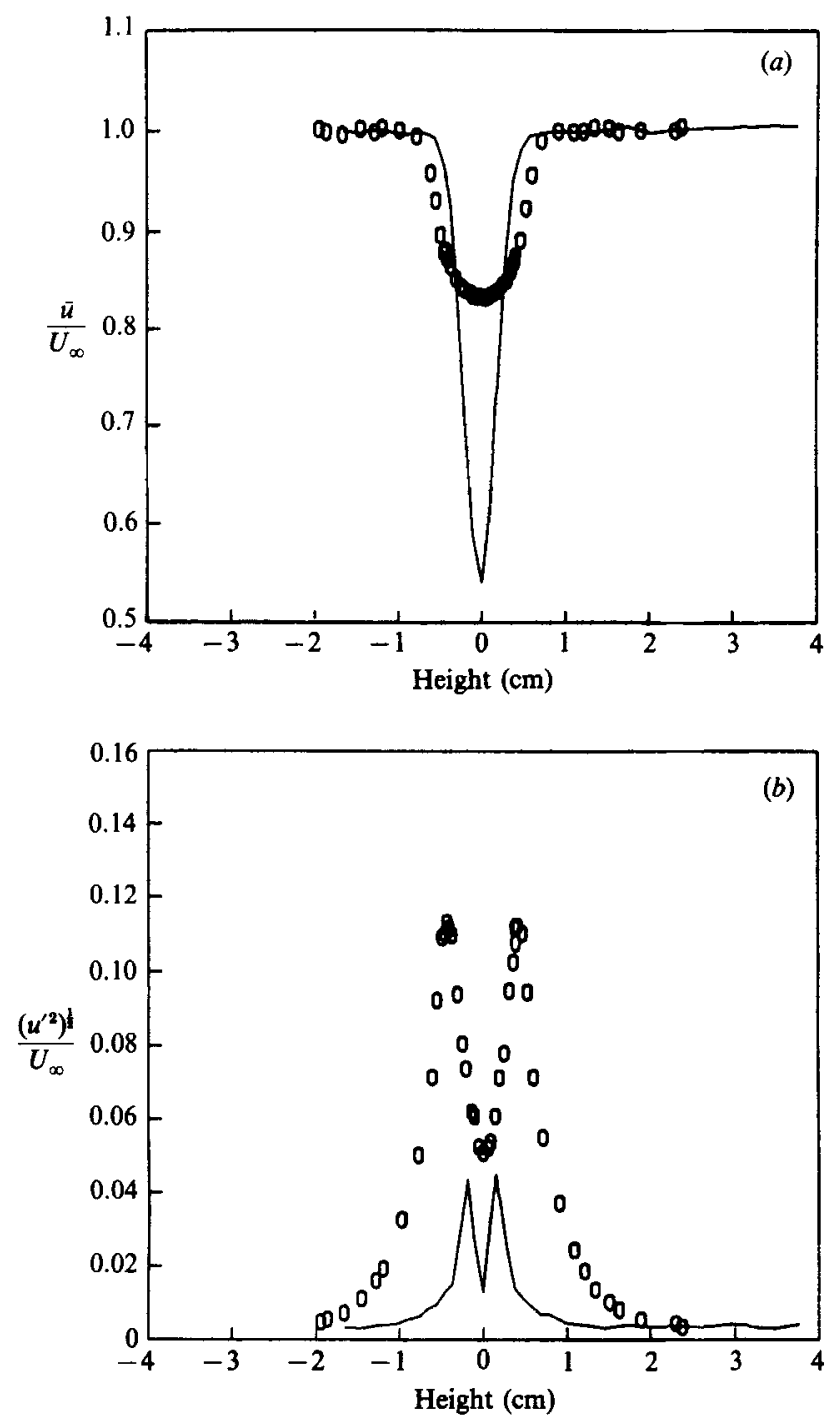

Figure 21. Velocity profiles of a locked case $(f=4.2 \mathrm{~Hz})(O)$ compared to the unforced case $(\longrightarrow)$ at $x / c=1:(a)$ mean velocity; (b) r.m.s. velocity.

frequency forced at a very low level) (figure $27 a$ ). As a second frequency $\left(f_{1}\right)$ is introduced with increasing power (figure $27 b-d$ ) the amplitude of the initial frequency decreases and becomes dominated by the second frequency. At the end, the initial frequency is barely visible in the flow. According to the definition of locking presented earlier, the flow is locked to $f_{1}$. If changes to the mean velocity profile are the key to the frequency selection process, it is possible that a linear stability analysis (which is known to be able to predict the initial vortex roll-up frequency (Freymuth; Ho \& Huang) in spite of the nonlinearity of the transition (roll-up) process) may provide some insight to the problem.

To establish the nature of the stability properties of the forced flows, a linear inviscid stability analysis was applied to mean velocity profiles measured at $x / c=$ 0.15 and at $x / c=1$. As was presented, there can be major changes in the mean 

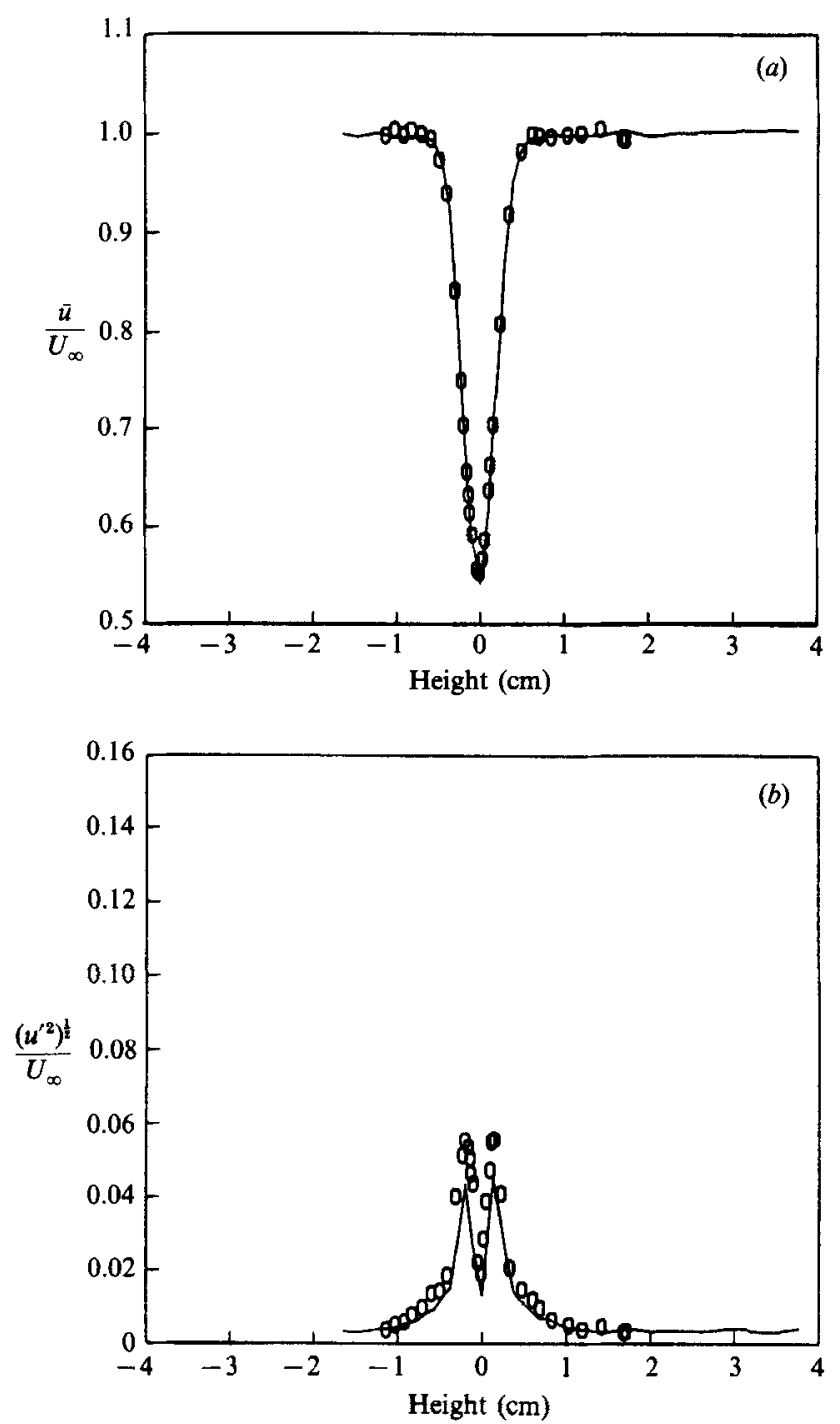

Figure 22. Velocity profiles of an unlocked case $(f=3.5 \mathrm{~Hz})(O)$ compared to the unforced case (-) at $x / c=1:(a)$ mean velocity; $(b)$ r.m.s. velocity.

velocity profile when the flow is forced which would imply that some change to the stability characteristics should occur. The inviscid stability of wakes and free shear layers has been examined experimentally by Sato \& Kuriki; Mattingly \& Criminale; Michalke (1965), and Monkewitz (1988). After comparing the results of the temporal analysis of Sato \& Kuriki to their own spatial analysis of a wake, Mattingly \& Criminale indicated that wake stability should best be considered from a spatial viewpoint. It is the spatial analysis that is applied to the current wake measurements. Following the procedure of conventional linearized stability calculations similar to that of Koochesfahani \& Frieler (1987) and Michalke, the basic flow $U$ is assumed to be two-dimensional and a function only of the cross-stream variable, $y$. The equations of motion and continuity are made non-dimensional by taking as units of velocity and length the free-stream velocity, $U_{\infty}$, and the wake half-width, $b$, where $u(y / b=1)=\frac{1}{2}\left(U_{\infty}+U_{\mathrm{c}}\right)$. 


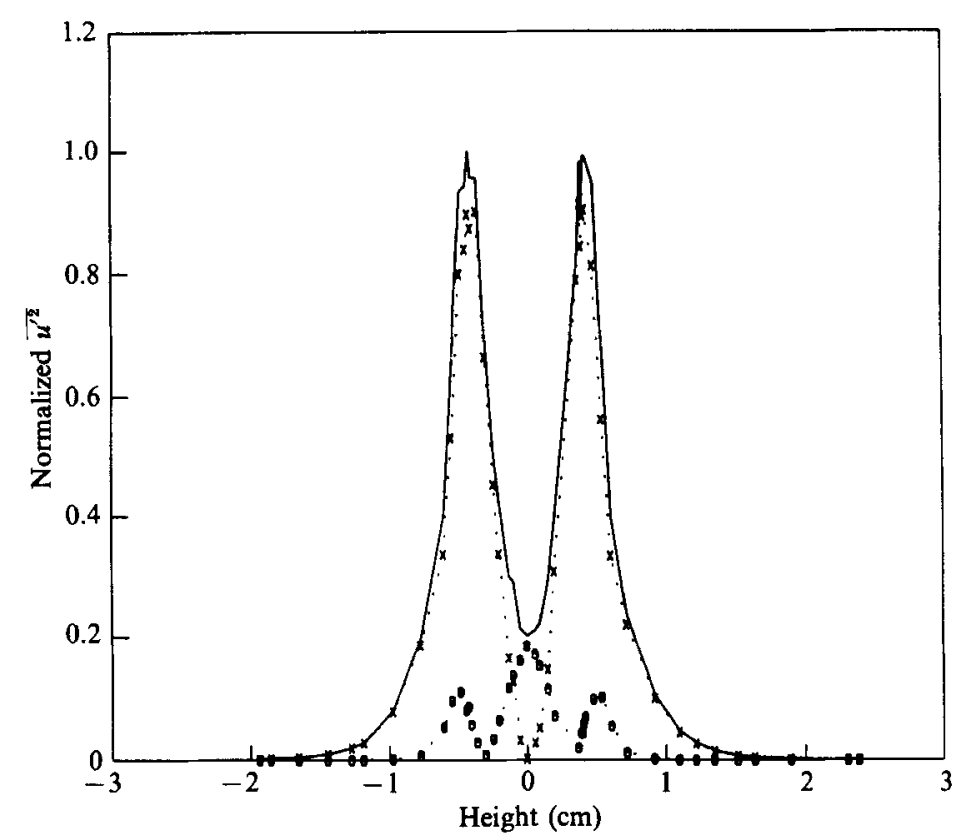

Figure 23. Bandwidth $\overline{u^{\prime 2}}$ profile for locked case at $x / c=1: \times$, first harmonic; $\mathrm{O}$, second harmonic.

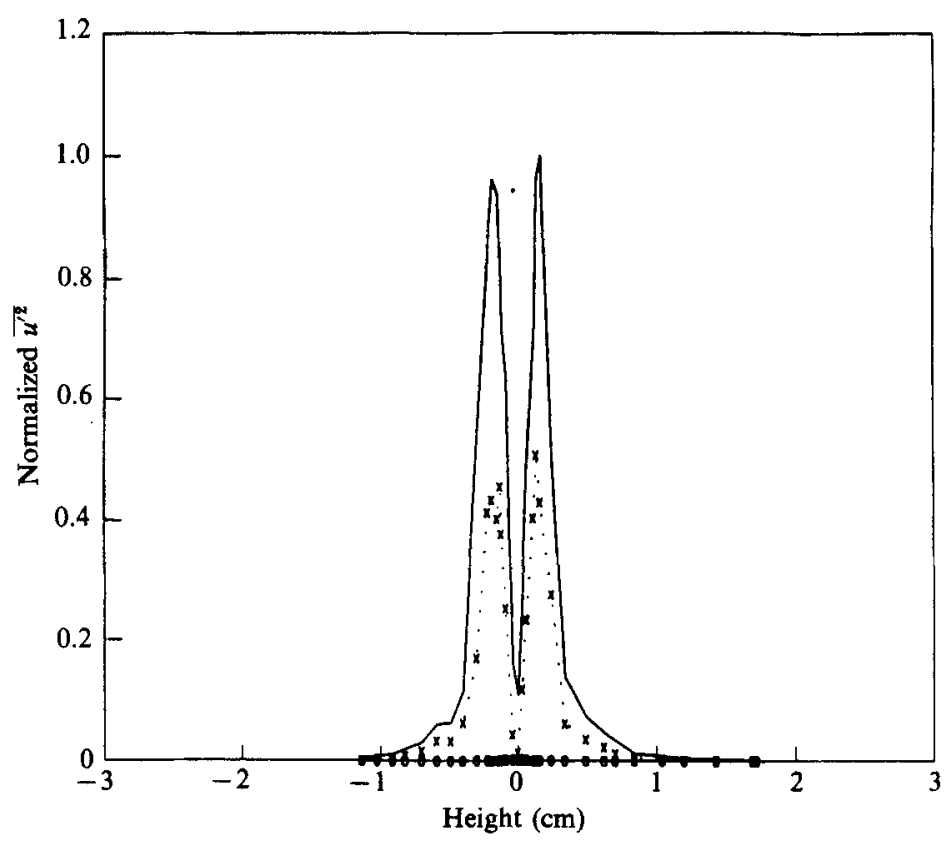

Frgure 24. Bandwidth $\overline{u^{2}}$ profile for unlocked case at $x / c=1: \times$, first harmonic ; $O$, second harmonic. 


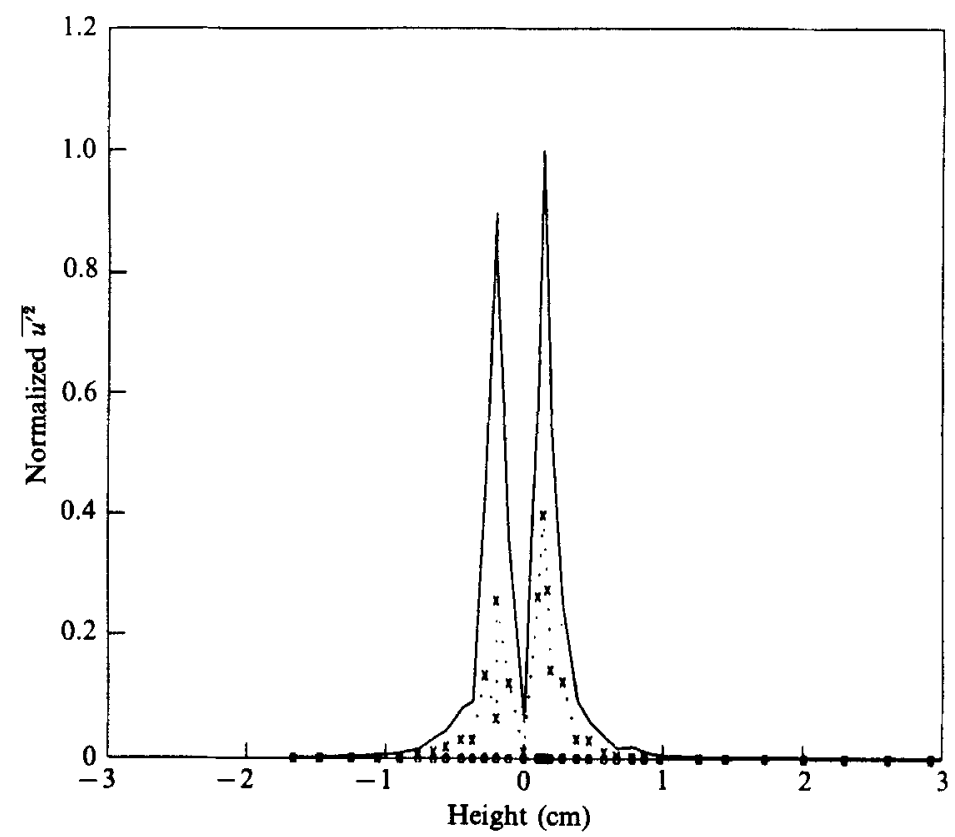

Figure 25. Bandwidth $\overline{u^{\prime 2}}$ profile for natural case at $x / c=1: \times$, first harmonic; $\mathrm{O}$, second harmonic.

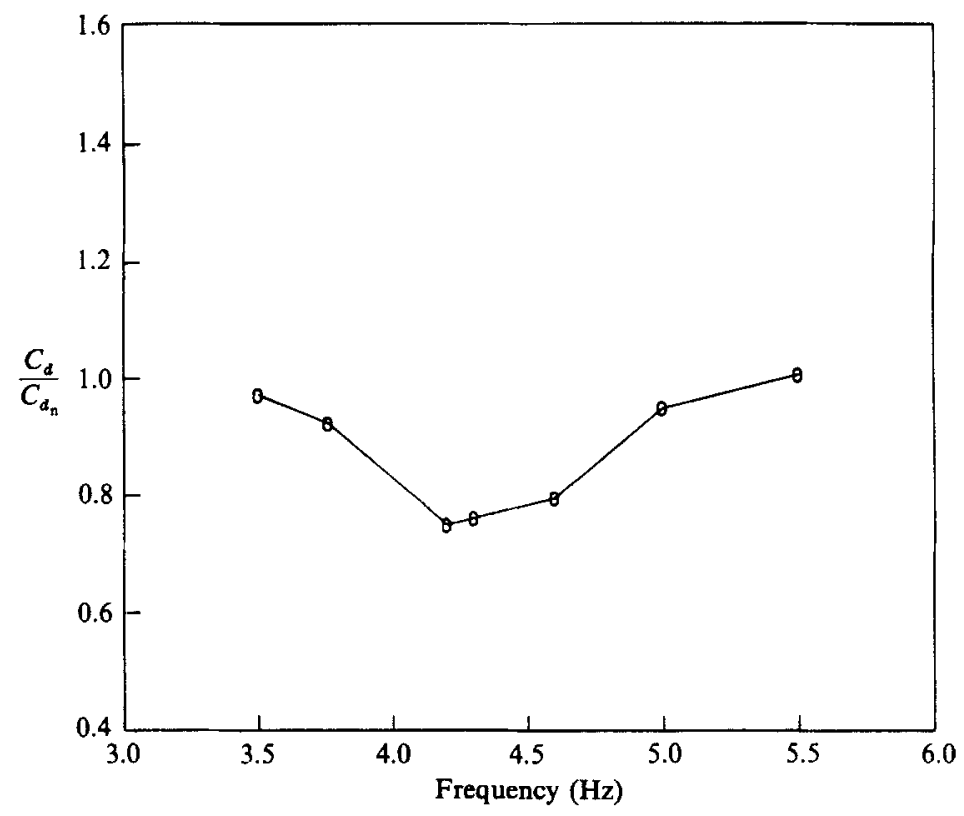

FIGURE 26. Drag coefficient as a function of forcing frequency relative to the unforced drag coefficient at $x / c=1$. 

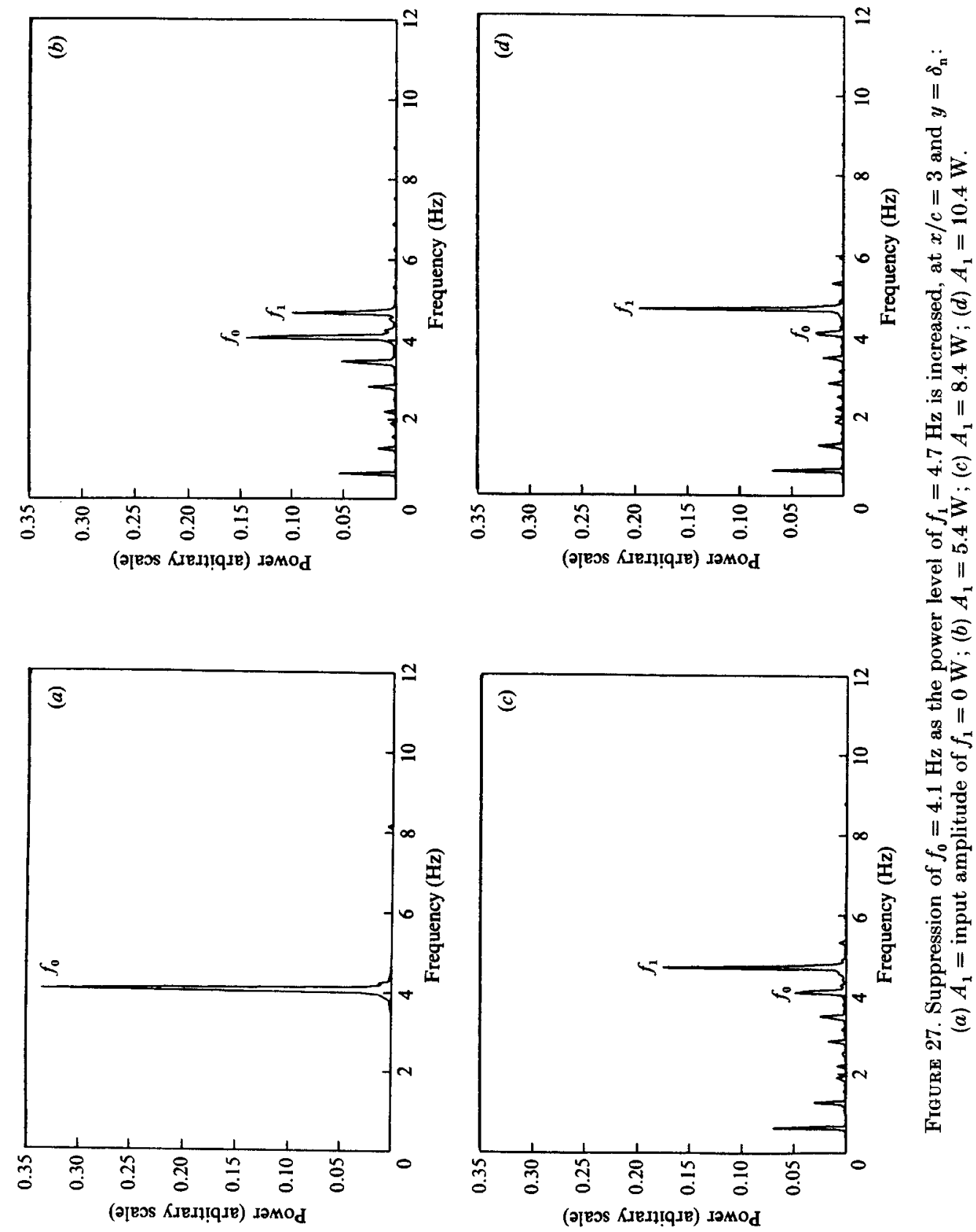
If the dimensional quantities are taken to be $X, Y$, and $T$, the dimensionless variables are then defined as $x=X / b, y=Y / b$, and $t=T U_{\infty} / b$. A disturbance stream function is written

$$
\psi(x, y, t)=\phi(y) \mathrm{e}^{\mathrm{i}(\alpha x-\beta t)},
$$

where $\alpha=\alpha_{\mathrm{r}}+\mathrm{i} \alpha_{\mathrm{i}}$ is the complex non-dimensional wavenumber, and $\beta$ is the nondimensional frequency, which is taken to be real for the spatial calculation. The perturbation velocities are related to the stream function by

$$
u=\operatorname{Re}\left\{\frac{\partial \psi}{\partial y}\right\}, \quad v=\operatorname{Re}\left\{-\frac{\partial \psi}{\partial x}\right\} .
$$

It is assumed that $u$ and $v$ are small enough to justify linearization of the equations of motion. For two-dimensional, inviscid, incompressible flow with uniform density and negligible buoyancy effects (i.e. the effect of gravity is ignored), the disturbance stream function satisfies the Rayleigh equation,

$$
\left(\phi^{\prime \prime}-\alpha^{2} \phi\right)\left(U-\frac{\beta}{\alpha}\right)-U^{\prime \prime} \phi=0,
$$

where a prime denotes differentiation with respect to $y$. This eigenvalue equation is solved using a 'shooting' technique subject to the boundary conditions

$$
\phi(y \rightarrow \pm \infty)=\mathrm{e}^{\mp \alpha y} .
$$

For a given $\beta$, equation (1) is integrated from both sides towards $y=0$. Matching $\phi$ and $\phi^{\prime}$ at the centreline $(y=0)$ yields the spatial growth rate, $-\alpha_{i}$, and the corresponding wavenumber, $\alpha_{r}$, of the unstable disturbance. The non-dimensional frequency, $\beta$, is related to real frequencies through the relation

$$
\beta=2 \pi f b / U_{\infty} \text {. }
$$

The wake mean velocity profiles were smoothed to fit either the curve

or

$$
\begin{gathered}
U(y)=1+\lambda \tanh (y)-W \mathrm{e}^{-k y^{2}} \\
U(y)=1+\lambda \tanh (y)-W \operatorname{sech}^{2}\left(\sigma y^{2}\right),
\end{gathered}
$$

where $y=\left(Y-Y_{0}\right) / b$ where $Y_{0}$ is the centreline height, $U(y)$ is the mean velocity profile scaled by the free-stream velocity, $U_{\infty}=\frac{1}{2}\left(U_{\text {top }}+U_{\text {bottom }}\right)$ where $U_{\text {top }}$ and $U_{\text {bottom }}$ are the mean velocities above and below the airfoil, $\lambda=$ $\left(U_{\text {top }}-U_{\text {bottom }}\right) /\left(U_{\text {top }}+U_{\text {bottom }}\right)$ (it was felt that forcing might create a shear in the profile, and thus separate top and bottom velocities were considered), and $W=$ $\left(U_{\infty}-U_{\mathrm{c}}\right) / U_{\infty}=$ the normalized wake deficit. The constants $k$ and $\sigma$ are set equal to In 2 and 0.88137 , respectively, so that $U(y=1)=\frac{1}{2}$. The hyperbolic secant was necessary for fitting the downstream profiles of wakes forced near the natural frequency, which tended to be more rounded in shape. For each profile, a best fit for $b$ was determined by minimizing the mean-square error between the experimental and analytical curves.

The results of the stability analysis are presented in the form of amplification curves where the amplification rate $\left(-\alpha_{1}\right)$ normalized by the maximum rate for the case is plotted as a function of frequency. The frequency axis (for $x / c=0.15$ results) is scaled so that the most-amplified frequency for the numerical results corresponds to the experimentally determined most-amplified frequency. The same factor is then used for all of the $x / c=0.15$ profiles (forced and unforced). This scaling can be justified if one considers the fact that the lengthscale used for the height 


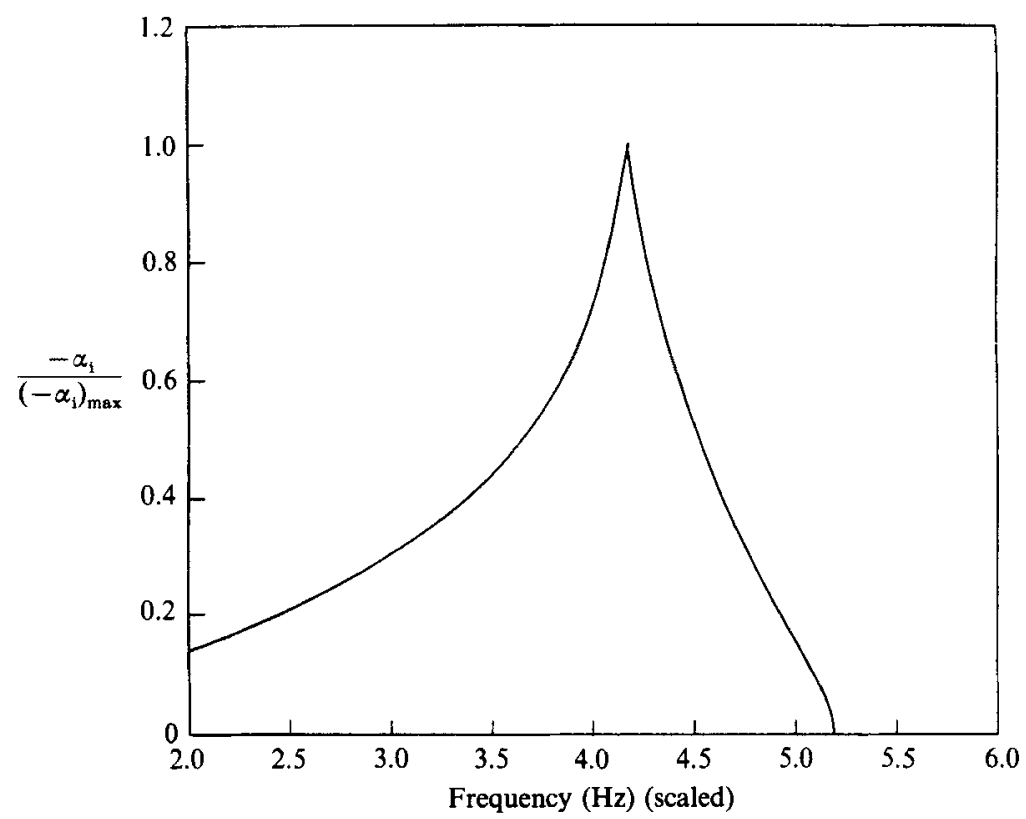

Figure 28. Linear stability amplification curve for the natural wake at $x / c=0.15$.

normalization (the half-wake width) has become a standard based on empirical results. From a physical viewpoint, another lengthscale (such as a vorticity thickness) may be more appropriate and might lead to more accurate results.

The first set of velocity profiles to be analysed were measured at the airfoil trailing edge $(x / c=0.15)$. The natural case and four forced cases (frequencies $=4.16,4.2$, 4.22 , and $4.3 \mathrm{~Hz}$ respectively) are considered. All of the forcing frequencies are near the centre of the locking range. The results for the natural (unforced) wake are presented in figure 28. The peak corresponds to the frequency $4.2 \mathrm{~Hz}$. Consider next the stability curves for the forced cases (figure 29). The peak frequencies are within $1 \%$ of the forcing frequencies for each respective case. The total shift in frequency represents $10 \%$ of the locking range at the given power level. In other words, the wake is adjusting itself through changes to the mean velocity profile such that it is more receptive to the forcing frequency, and thus less receptive to other frequencies. This effect is most likely the cause for the suppression of one frequency in the presence of a second stronger frequency. An error analysis confirmed that changes in the wake width greater than the resolution of the fitting technique must occur before significant shifts in the peak frequency can be observed.

The amplification curves for the natural wake and five forced cases (forcing frequencies $3.5,4.2,4.3,4.6$, and $5.5 \mathrm{~Hz})$ at a station further downstream $(x / c=1)$ are plotted in figure 30 . Owing to the lack of a sharp peak near the maximum of each curve, the most-amplified frequency was not determined. Thus the frequency axis is not scaled as in figure 29. The unscaled nature of the plot is misleading in that the peak frequencies appear to be higher than the $x / c=0.15$ results. It is the relative changes between the strongly and weakly forced flows which is emphasized here. The two cases outside of the locking range $(3.5 \mathrm{~Hz}, 5.5 \mathrm{~Hz})$ have stability characteristics similar to the unforced wake. As the forcing frequency approaches the centre of the experimentally determined receptivity curve, the range of amplified frequencies narrows and shifts towards lower frequencies. In Mattingly \& Criminale, the 


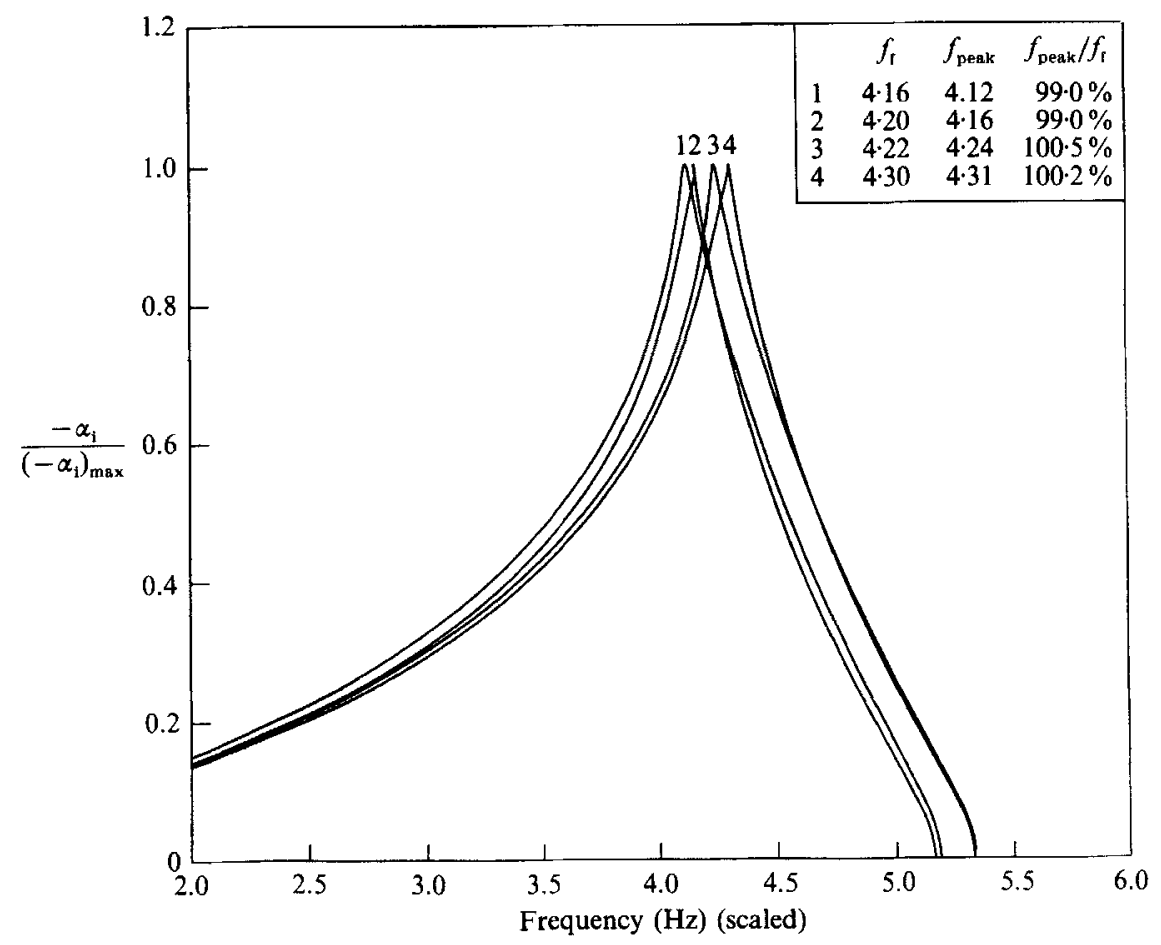

Frgure 29. Linear stability amplification curves for four forced wakes at $x / c=0.15$.

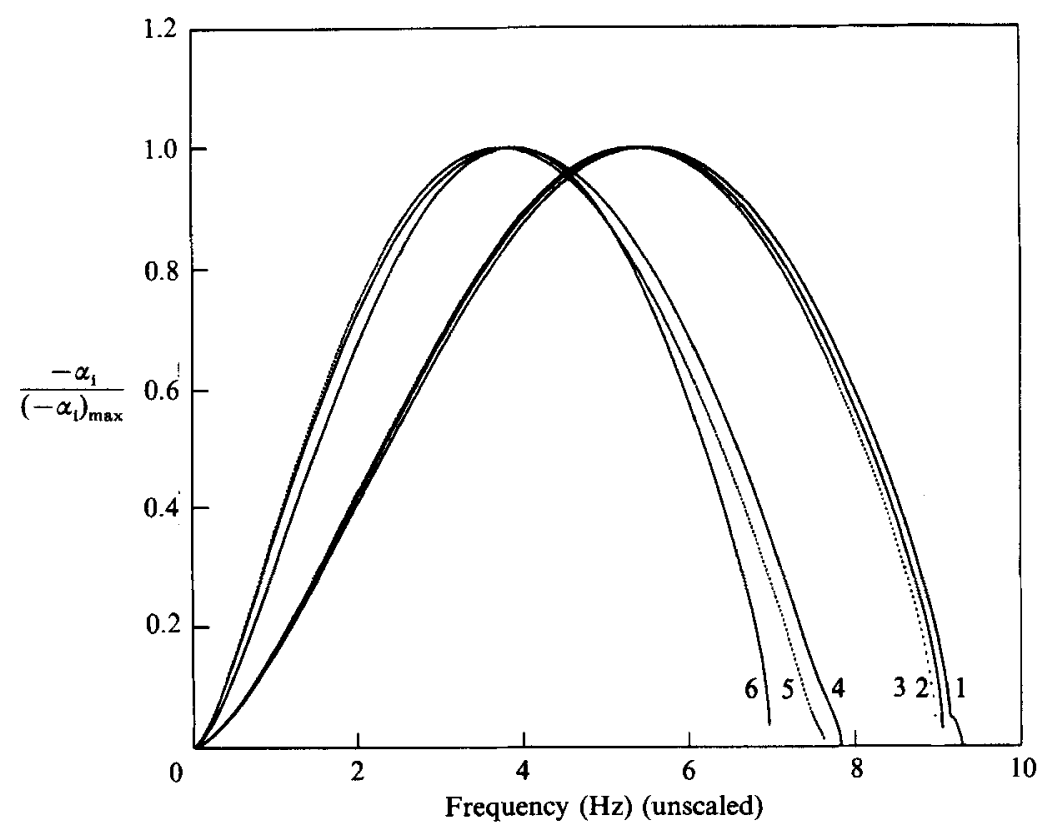

Figure 30. Linear stability amplification curve for the natural wake and five forced wakes at $x / c=1$ : curve 1 , unforced; $2,5.5 \mathrm{~Hz} ; 3,3.5 \mathrm{~Hz} ; 4,4.3 \mathrm{~Hz} ; 5,4.2 \mathrm{~Hz} ; 6,4.6 \mathrm{~Hz}$. 


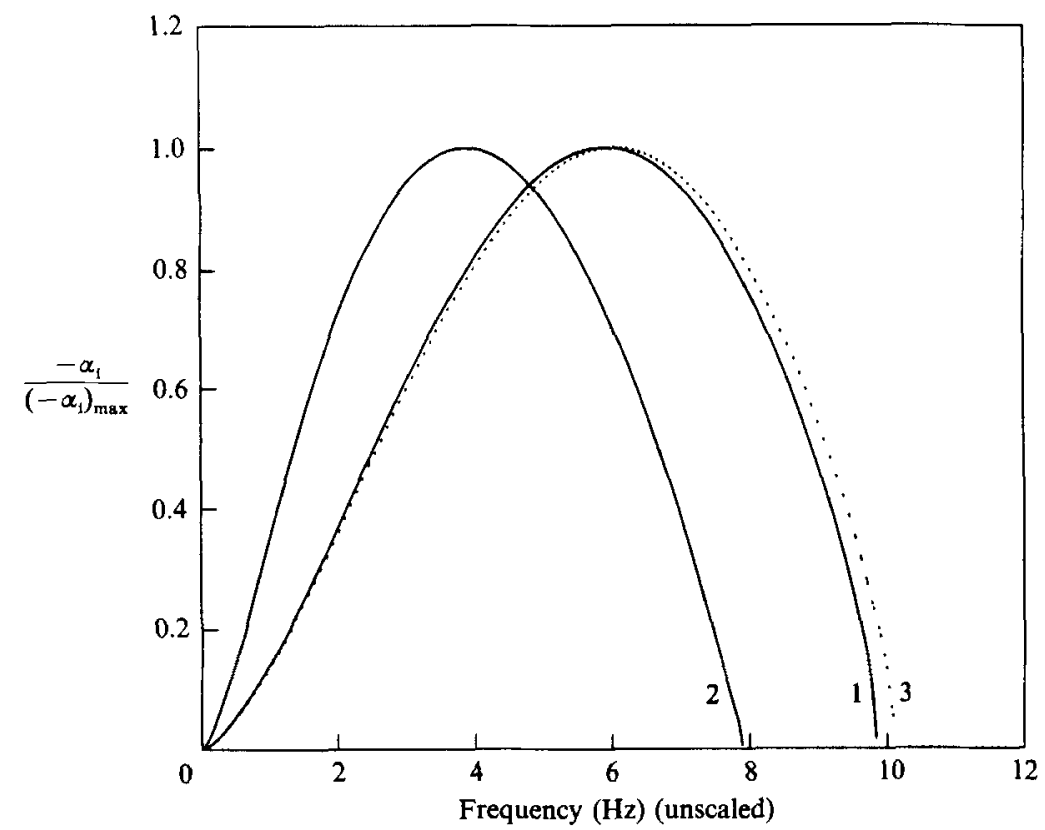

FIaURe 31. Linear stability amplification curves for (1) natural, (2) asymmetric, and (3) symmetric caes at $x / c=1$.

maximum amplification rate decreases as the downstream distance increases. This decrease was observed particularly for forcing near the most-amplified frequency (prior to scaling), given the shift to lower frequencies and reduced amplification rate for highly amplified frequencies, it appears that strong forcing produces a more rapid transition in the wake.

Evaluation of $\phi$ and $\phi^{\prime}$ at the centreline indicates that the most-amplified mode detected is the sinuous mode, $\phi(0)=1$ and $\phi^{\prime}(0)=0$ (as described by Wygnanski in several references). For the $x / c=0.15$ measurements, the varicose mode was observed as a pure mode; however, the amplification rates were substantially reduced compared to the sinuous mode.

The linear stability analysis applied to the mean profiles at $x / c=1$ for the cancellation/enhancement cases (figure 31) shows that the flow returns to a state similar to the unforced case when the natural frequency is forced symmetrically. Thus, a near complete cancellation is observed.

\section{Concluding remarks}

We have shown that for effective forcing of a shear flow, it is necessary to establish the receptivity range of the system. This becomes important when developing a technique for examining the effect of modifying initial conditions on the dynamics of the wake.

In this paper we have demonstrated the effectiveness of the strip-heater technique for enhancing or cancelling waves in the wake through variation of the phase difference between the waves. A momentum balance analysis of the forced wake at its maximum amplified frequency shows a drag reduction of 10 to $30 \%$. Although we could not establish a reason for this drag reduction, we conjecture that a thrust 
mechanism similar to that of a flapping airfoil is generated by the variations of the boundary-layer displacement thickness when forced by the strip heaters.

The frequency selection process in the wake is addressed by assuming that this process is greatly influenced in the initial stages of the wake. Accordingly, by conducting a linear stability analysis, a correspondence between the frequency selection process and changes to the mean velocity profile is established. It was found that the mean velocity profile adapts itself to become more receptive to the frequency of the forced oscillation. It is interesting to note that a linear stability analysis based on the mean velocity profile reveals this connection in spite of the nonlinearity of the transition process. However, the analysis is incapable of explaining the exact mechanisms that are responsible for the mean velocity profile changes. Further research to be performed with regard to the forced wake would encompass a study of the entrainment and vorticity concentration in the wake.

This paper is based on the work for the Ph.D. thesis of K. Stuber which was carried out at the University of California, San Diego. The work was sponsored by the Defense Advanced Research Projects Agency-ACMP through the URI program under contract number DARPA/ONR N00014-86-K-0758. The assistance of INLS/UCSD is also acknowledged. The authors would like to express our gratitude to Professor M. M. Koochesfahani for his assistance in implementing the linear stability program.

\section{REFERENCES}

Browand, F. K. 1966 An experimental investigation of the instability of an incompressible, separated shear layer. J. Fluid Mech. 26, 281-307.

Freymuth, P. 1966 On transition in a separated laminar boundary layer. J. Fluid Mech. 25, 683-704.

GhaRIB, M. 1987 Response of the cavity shear layer oscillations to external forcing. AIAAJ.25, 43-47.

Ho, C.-M. \& HuANG, L.-S. 1982 Subharmonics and vortex merging in mixing layers. J. Fluid Mech. 119, 443-473.

Koochesfahani, M. M. 1987 Vortical patterns in the wake of an oscillating airfoil. AIAA-870111.

Koochesfahani, M. M. \& Frieler, C. E. 1987 Inviscid instability characteristics of free shear layers with non-uniform density. $A I A A-87-0047$.

Liepmann, H. W., Brown, G. L. \& Nosenchuck, D. M. 1982 Control of laminar-instability waves using a new technique. J. Fluid Mech. 118, 187-200.

Liepmann, H. W. \& Nosenchuck, D. M. 1982 Active control of laminar-turbulent transition. J. Fluid Mech. 118, 201-204.

Mattingly, G. E. \& Criminale, W. O. 1972 The stability of an incompressible two-dimensional wake. J. Fluid Mech. 51, 233-272.

Michalke, A. 1965 On spatially growing disturbances in an inviscid shear layer. J. Fluid Mech. 23, 521-544.

Miksad, R. W. 1972 Experiments on the nonlinear stages of free shear layer transition. J. Fluid Mech. 56, 695-719.

Mrksad, R. W. 1973 Experiments on nonlinear interactions in the transition of a free shear layer. J. Fluid Mech. 59, 1-21.

Monkewitz, P. A. 1988 The absolute and convective nature of instability in two dimensional wakes at low Reynolds numbers. Phys. Fluids 31, 999-1006.

Oster, D. \& Wygnanski, I. 1982 The forced mixing layer between parallel streams. J. Fluid Mech. 123, 91-130. 
RoвERTs, F. A. 1985 Effects of a periodic disturbance on structure and mixing in turbulent shear layers and wakes. Ph.D. thesis, California Institute of Technology, Pasadena, CA.

RoвEY, H. F. 1986 On the nature of oblique instability waves in boundary layer transition. Ph.D. thesis, California Institute of Technology, Pasadena, CA.

SATO, H. 1970 An experimental study of non-linear interaction of velocity fluctuations in the transition region of a two-dimensional wake. J. Fluid Mech. 44, 741-765.

Sato, H. \& KuRIKI, K. 1961 The mechanism of transition in the wake of a thin flat plate placed parallel to uniform flow. J. Fluid Mech. 11, 321-352.

SchUbaUtR, G. B. \& Skramstad, H. K. 1947 Laminar boundary layer oscillations and stability of laminar flow. J. Aero. Sci. 14, 68-78.

STUBER, K. 1988 An experimental investigation of the effect of external forcing on the wake of a thin airfoil. Ph.D. thesis, University of California, San Diego, La Jolla, CA.

Wygnanski, I., Champagne, F. \& Marasli, B. 1986 On the large-scale structures in twodimensional, small-deficit, turbulent wakes. J. Fluid Mech. 168, 31-71.

Wygnanski, I., Oster, D., Fiedler, H. \& Dziomba, B. 1979 On the perseverance of a quasi-twodimensional eddy-structure in a turbulent mixing layer. J. Fluid Mech. 93, 325-335.

ZAMan, K. B. M. Q. \& Hussain, A. K. M. F. 1981 Turbulence suppression in free shear flows by controlled excitation. J. Fluid Mech. 103, 133-159. 